\title{
CaCBL1 Acts as a Positive Regulator in Pepper Response to Ralstonia solanacearum
}

\author{
Lei Shen, ${ }^{1,2,3}$ Sheng Yang, ${ }^{1,2,3}$ Feng Yang, ${ }^{1,2,3}$ Deyi Guan, ${ }^{1,2,3}$ and Shuilin $\mathrm{He}^{1,2,3, \dagger}$ \\ ${ }^{1}$ Key Laboratory of Applied Genetics of Universities in Fujian Province, Fujian Agriculture and Forestry University, Fuzhou, \\ Fujian 350002, PR China \\ ${ }^{2}$ National Education Ministry Key Laboratory of Plant Genetic Improvement and Comprehensive Utilization, Fujian Agriculture \\ and Forestry University, Fuzhou, Fujian 350002, PR China \\ ${ }^{3}$ Agricultural College, Fujian Agriculture and Forestry University, Fuzhou, Fujian 350002, PR China \\ Accepted 23 March 2020.
}

\begin{abstract}
Bacterial wilt caused by Ralstonia solanacearum is an important disease of pepper (Capsicum annuum), an economically important solanaceous vegetable worldwide, in particular, under high temperature (HT) conditions. However, the molecular mechanism underlying pepper immunity against bacterial wilt remains poorly understood. Herein, CaCBL1, a putative calcineurin B-like protein, was functionally characterized in the pepper response to $R$. solanacearum inoculation (RSI) under HT (RSI/HT). CaCBL1 was significantly upregulated by RSI at room temperature (RSI/RT), HT, or RSI/HT. CaCBL1-GFP fused protein targeted to whole epidermal cells of Nicotiana benthamiana when transiently overexpressed. $C a C B L 1$ silencing by virus-induced gene silencing significantly enhanced pepper susceptibility to RSI under RT or HT, while its transient overexpression triggered hypersensitive response mimic cell death and upregulation of immunity-associated marker genes, including CabZIP63, CaWRKY40, and CaCDPK15, the positive regulators in the pepper response to RSI or HT found in our previous studies. In addition, by chromatin immunoprecipitation PCR and electrophoretic mobility shift assay, $C a C B L 1$ was found to be directly targeted by CaWRKY40, although not by CaWRKY27 or CaWRKY58, via the W-box-2 within its promoter, and its transcription was found to be downregulated by silencing of $\mathrm{CaWRKY40}$ while it was enhanced by its transient overexpression. These results suggest that CaCBL1 acts as a positive regulator in pepper immunity against $R$. solanacearum infection, constituting a positive feedback loop with $\mathrm{CaWRKY40.}$
\end{abstract}

Keywords: CaCBL1, CaWRKY40, R. solanacearum, high temperature, pepper

In their natural habitats, plants are frequently challenged by threats from various pathogens. To protect themselves, plants perceive pathogen-associated molecular patterns (PAMPs) and

${ }^{\dagger}$ Corresponding author: S. He; shlhe201304@aliyun.com

Funding: This work was supported by grants from the National Natural Science Foundation of China (31572136) and the Scientific Research Foundation of the Graduate School of Fujian Agriculture and Forestry University (324-1122yb045).

*The $\boldsymbol{e}$-Xtra logo stands for "electronic extra" and indicates that four supplementary figures and one supplementary table are published online.

The author(s) declare no conflict of interest.

๑) 2020 The American Phytopathological Society effectors of pathogens via pattern recognition receptors and resistance proteins, leading to PAMP-triggered immunity (PTI) and effector-triggered immunity (ETI) (Hein et al. 2009; Jones and Dangl 2006). PTI is conserved across different plant species and can impede the development of pathogens but is not sufficient for disease resistance, while ETI is specific to the pathogen strain or race and is generally associated with programmed cell death. The activated immune responses in ETI occur more quickly quick and are more prolonged and more robust than those in PTI (Tsuda and Katagiri 2010; Tsuda et al. 2009). Despite these distinct features between PTI and ETI, defense signaling components include oxidative burst, $\mathrm{Ca}^{2+}$ influx, production of phytohormones such as salicylic acid (SA), jasmonic acid (JA), and ethylene (ET), and mitogen-activated protein kinase (MAPK) activation have been frequently found to be shared by PTI and ETI with different timing and magnitude (Tsuda and Katagiri 2010). The defense signaling initiated upon the perception of PAMPs and effectors by their corresponding receptors in plants are transmitted from cytoplasm into the nucleus, in which they are integrated and translated into appropriate transcriptional outputs with the action of various transcription factors (TFs) (Buscaill and Rivas 2014; Moore et al. 2011). Some of these TFs form positive or negative feedback loops with their upstream signaling components to produce appropriate immune reaction (Guan et al. 2018; Shen et al. 2016b). However, the signaling components in PTI and ETI have not been fully characterized, and how they are related to downstream TFs remains to elucidated.

Calcium is a ubiquitously intracellular secondary messenger that is involved in plant response to a variety of biotic and abiotic stresses. The fluctuation of calcium ion concentrations in the cytoplasm varies, dependent on the nature of stress, and possibly contributes to the specificity of response. The $\mathrm{Ca}^{2+}$ signal is decoded and transmitted downstream by $\mathrm{Ca}^{2+}$ sensors including calmodulins (CaMs), calmodulin-like proteins (CMLs), calcineurin B-like proteins (CBLs), and calciumdependent protein kinases (CPKs) (Kudla et al. 2010). CBL proteins collectively possess three or four EF-hand domains for $\mathrm{Ca}^{2+}$ ion binding (Zhang et al. 2011), mediate calcium signaling by binding to and activating protein kinases named CBL interacting protein kinases (CIPKs) (Luan 2009). CBLs are encoded by a gene family with 10 members in Arabidopsis and rice (Kolukisaoglu et al. 2004), seven in canola (Zhang et al. 2014a), 12 in maize (Zhang et al. 2016), 15 in soybean (Zeng et al. 2017), 19 in turnip (Yin et al. 2017), and eight in grapevine (Xi et al. 2017). Phylogenetic analysis showed that CBLs fall into six different groups (Mohanta et al. 2015). Members of this family have been implicated by interacting with 
CIPKs in the plant response to salt stress (Deng et al. 2013; Kim et al. 2007; Liu et al. 2015b; Luo et al. 2017; Tang et al. 2014; Wang et al. 2007), drought stress (Cheong et al. 2007; Zhu et al. 2016), cold stress (Deng et al. 2013; Kudla et al. 1999; Zhou et al. 2016), and pathogen attack (de la Torre et al. 2013; Zhang et al. 2014b). However, the role of the majority of CBLs in plant response to high temperature (HT) and to simultaneous HT and pathogen attack remains elusive.

WRKY TFs, constituting one of the largest plant TF families, are characterized by their one or two conserved WRKY domains containing the sequence WRKYGQK and their bindings to the highly conserved $\mathrm{W}$ boxes [TTGAC $(\mathrm{C} / \mathrm{T})]$ present in the promoter regions of their target genes (Cheong et al. 2007; Zhu et al. 2016). Members of this family have been implicated in plant immunity (Bhattacharjee et al. 2013; Bhattarai et al. 2010; Birkenbihl et al. 2012; Chen et al. 2013; Dang et al. 2013; Hu et al. 2012; Kalde et al. 2003; Pandey and Somssich 2009) and response to abiotic stresses including heat stress (Cai et al. 2015; Dang et al. 2013; Li et al. 2010, 2011), and some members, such as AtWRKY25 (Li et al. 2009), AtWRKY26 (Li et al. 2011), AtWRKY33 (Li et al. 2011), and AtWRKY39 (Moore et al. 2011) in Arabidopsis, CaWRKY6 (Cai et al. 2015) and CaWRKY40 (Dang et al. 2013) in pepper, act as convergence sites in plant response to pathogen and heat stress, but the roles of WRKY TFs in plant response to simultaneous pathogen infection and heat stress remain elusive. In addition, except for a few examples such as AtWRKY33, which was directly activated by MAPK cascades, and HvWRKY1 and HvWRKY2, which were recognized and regulated by immune receptors (Tsuda and Somssich 2015). However, the connections between the majority of WRKY TFs and their upstream signaling components have not been well-characterized. As a single WRKY might regulate the transcription of hundreds of target genes in a promoter-context dependent manner (Birkenbihl et al. 2017; Liu et al. 2015a), some of the target genes of WRKY TFs might encode their upstream regulatory components (Birkenbihl et al. 2017; Liu et al. 2015a). The functional characterization of the target genes of WRKY TFs can therefore provide a novel approach to identify the upstream regulatory nodes and new insight into the underlying mechanism of biological process mediated by these WRKY TFs.

Pepper is an agriculturally important solanaceous vegetable worldwide, and bacterial wilt caused by $R$. solanacearum is one of the most important diseases of pepper (Lafortune et al. 2005). However, the molecular details of how pepper responds to attack of $R$. solanacearum remain poorly understood. Our previous studies showed that CaCDPK15 (Shen et al. 2016b), CaWRKY6 (Cai et al. 2015), CaWRKY40 (Dang et al. 2013), and CabZIP63 (Shen et al. 2016a) play important roles in pepper immunity or tolerance to HT, in which CaWRKY4O is directly regulated by CaWRKY6 and CabZIP63 and indirectly by CaCDPK15 and their corresponding genes are in turn regulated by CaWRKY40. In the present study, we provided evidence that $C a C B L 1$ is directly transcriptionally upregulated by CaWRKY40 during pepper response to $R$. solanacearum inoculation (RSI) or HT and it acts as a positive regulator in pepper immunity under HT by modulating the transcription of CaWRKY40, CabZIP63 and CaCDPK15.

\section{RESULTS}

\section{Cloning and sequence analysis of $C a C B L 1$ and its direct targeting by CaWRKY40.}

Our previous study indicated that CaWRKY40 acts as a positive regulator in pepper response to both RSI and HT stress. To elucidate the underlying mechanism, the possible DNA binding sites of CaWRKY40 were searched by chromatin immunoprecipitation sequencing (ChIP-seq). Chromatins were isolated from 35S:CaWRKY40-Flag transiently overexpressing pepper leaves and were then sheared into fragments of 300 to $500 \mathrm{bp}$ in length and were further immunoprecipitated by anti-Flag antibody. The recovered DNA was subjected to sequencing (data not shown). A putative DNA binding site was found in the promoter of a putative CBL protein-encoding gene. To further assay its possible function signaling related to CaWRKY40, the corresponding full-length open reading frame (ORF) of this putative CBL was amplified by PCR, with the cDNA library of CM334 as template, using a specific primer pair. Within the deduced amino acid sequence, there are three EF-hand $\mathrm{Ca}^{2+}$ binding domains, which are conserved in $\mathrm{Ca}^{2+}$ binding proteins including CDPKs, CBLs, CMLs, and CaMs (Luan 2009) (Fig. 1A). The putative CBL shared $95,95,82,82,81,81,80,80$, 77 , and $73 \%$ sequence identities to its orthologs in Solanum tuberosum (CBL1), Solanum lycopersicum (CBL1), Populus trichocarpa (CBL1), Gossypium raimondii (CBL1), Populus euphratica (CBL9), Vitis vinifera (CBL1), Citrus sinensis $(C B L 1)$, Nicotiana tabacum (CBL1), Arabidopsis thalina $(C B L 1)$ and Oryza sativa Japonica Group (CBL1), respectively (Fig. 1B). We designated this CBL as CaCBL1 (XP_016551217.1). In addition, its promoter region of 2,000 bp in length was also identified and the cis elements within the region were assayed using PlantCARE online tools. The stress or pathogen-responsive cis elements such as the heat-shock and ETresponsive elements and the TCA element involved in SA responsiveness as well as two $\mathrm{W}$ boxes were found (Fig. 1C), implying the possible involvement of $\mathrm{CaCBL} 1$ in pepper response to pathogen infection or abiotic stresses. Noticeably, $C a C B L 1$ in pepper has not been functionally characterized, to date.

As two $\mathrm{W}$ boxes were found in the promoter region of $C a C B L 1$ (Fig. 2A), and CaCBL1 was found by ChIP-seq to be a potential target gene of CaWRKY40 (data not shown), it can be speculated that $C a C B L 1$ might be targeted by CaWRKY40. We tested this possibility by ChIP-PCR. Agrobacterium sp. strain GV3101 containing the 35S:CaWRKY40-Flag construct was infiltrated into pepper leaves, which were harvested at $48 \mathrm{~h}$ postinfiltration (hpi) for chromatin isolation. Western blot analysis was performed to confirm the expression of $35 \mathrm{~S}$ : CaWRKY40-Flag in pepper leaves (Fig. 2B). The chromatin was isolated and was ultrasonically sheared into 300- to 500-bp fragments, which were immunoprecipitated with anti-Flag antibody, and the acquired recovered DNA was used as template for PCR with primer pairs $\mathrm{P} 1$ and $\mathrm{P} 2$ of the fragment containing the two $\mathrm{W}$ boxes or the control primer pair of the fragment containing no $\mathrm{W}$ box in the promoter region of $C a C B L 1$. The result showed that only P2 clearly produced product (Fig. 2C; Supplementary Table $\mathrm{S} 1$ ), suggesting that $C a C B L 1$ is targeted, probably via the W-box-2 by CaWRKY40. To test if the binding of CaWRKY40 to the promoter of $C a C B L 1$ is selective or specific, CaWRKY27 and CaWRKY58, two WRKY TFs that have been implicated in pepper immunity against RSI by our previous studies (Dang et al. 2014; Wang et al. 2013), were selected to study their possible targeting to $C a C B L 1$ by ChIP-PCR. The expression of CaWRKY27 and CaWRKY58 at the transcriptional and posttranscriptional levels were detected by quantitative real-time PCR (qRT-PCR) and Western blot (Supplementary Fig. S1A and B), respectively. The results showed that neither of the two WRKY TFs bound to the two $\mathrm{W}$ boxes in the promoter of $C a C B L 1$, suggesting that the targeting of CaWRKY40 to CaCBL1 is selective (Supplementary Fig. S1C).

To confirm that $C a C B L 1$ is targeted by CaWRKY40, an electrophoretic mobility shift assay (EMSA) was performed to investigate the binding of CaWRKY40 to CaCBL1 promoter in vitro. For EMSA, the core amino acid sequence WRKYGQK of CaWRKY4O was mutated into WRKYGMK 


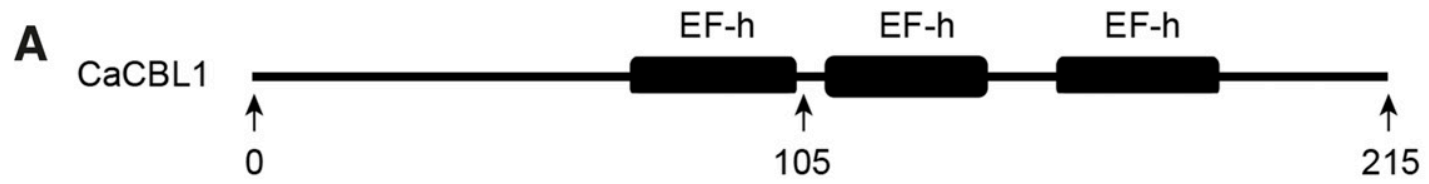

B
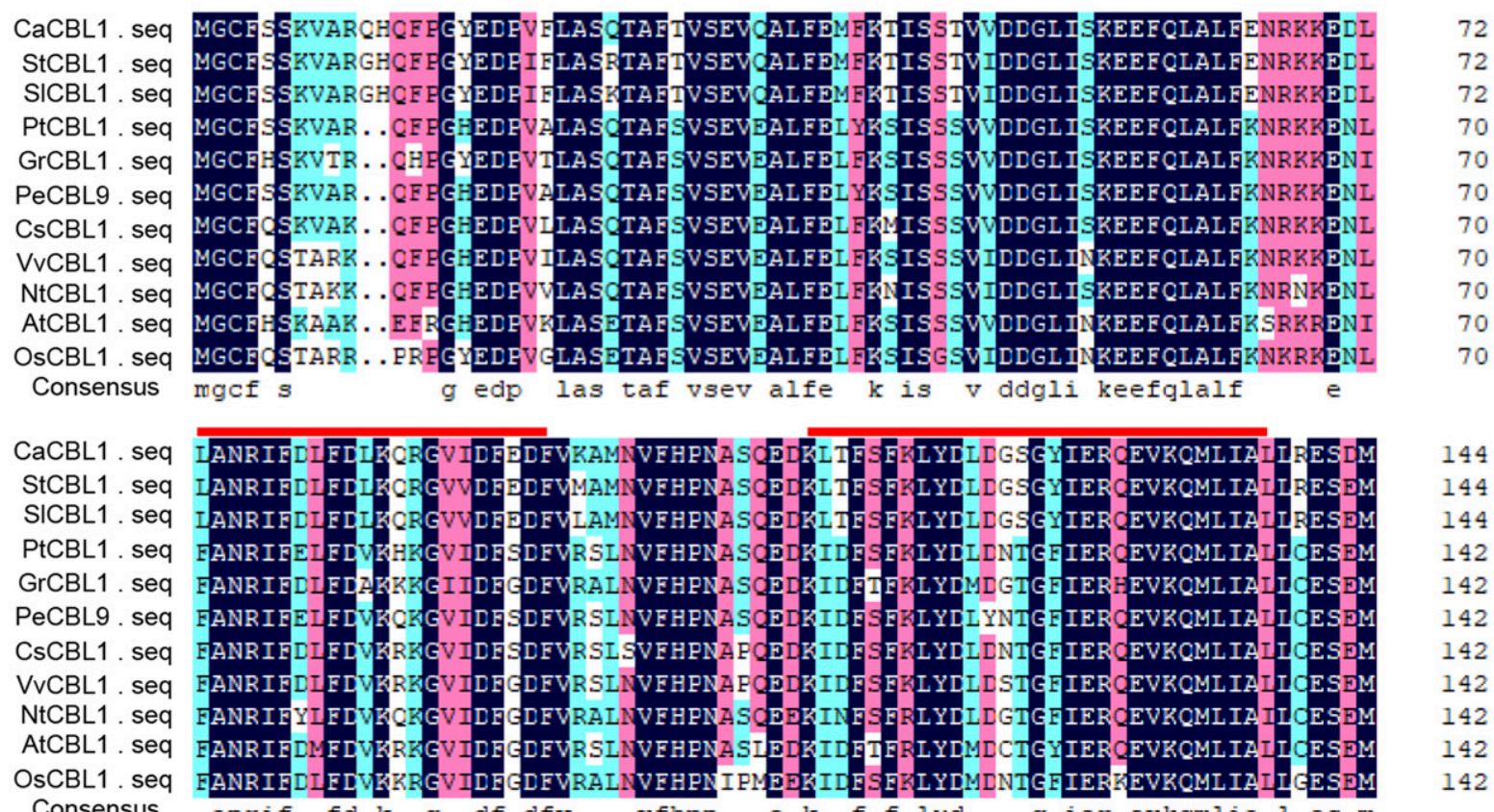

Consensus

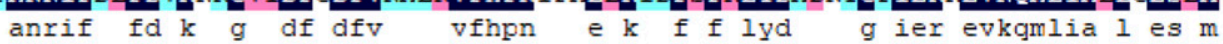

CaCBL1. seq KIZDETIEIILDKT SEALZNDDGKIDKSER̈CSFVGRNPSLLRIMTIEYLRDVTTTFPSFVEHSEVDEVA 214

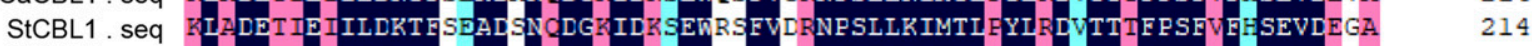
SICBL1. seq KIZDETIEIILDKT FSEADSNODGKIDRSERRSFVDRNPSLLRIMTIFYIRDVTTTFPSFVFHSEVDEGA 214 PtCBL1. seq KIZDETVEIILDKT FLDADVNRDGKIDKSEЙEN FVCRNPSLLRIMTLEYLADITTTFPSFVENSEVDEIA 212

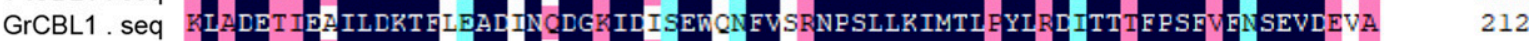

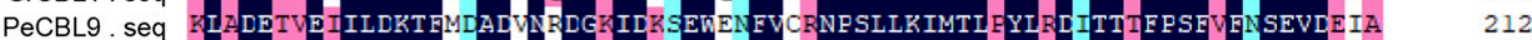

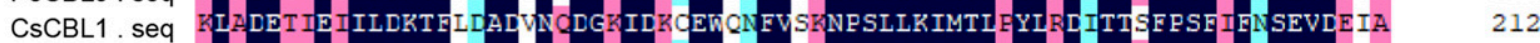

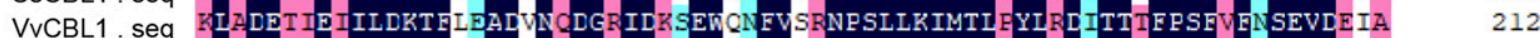

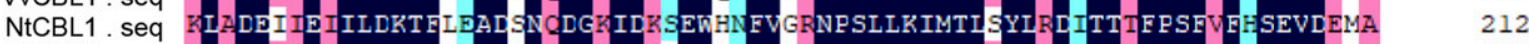
AtCBL1. seq KLZDETIBIILDKT FEDADVNODGKIDRLEЙSDFVNKNPSLLKIMTIFYIRDITTTFPSFVFHSEVDEIA 212

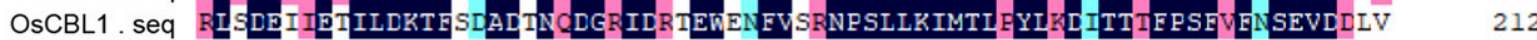

Consensus

1 de e ildktf ad $\mathrm{n}$ dg id ew fv npsllkimtl yl d tt fpsf $\mathrm{f}$ sevd

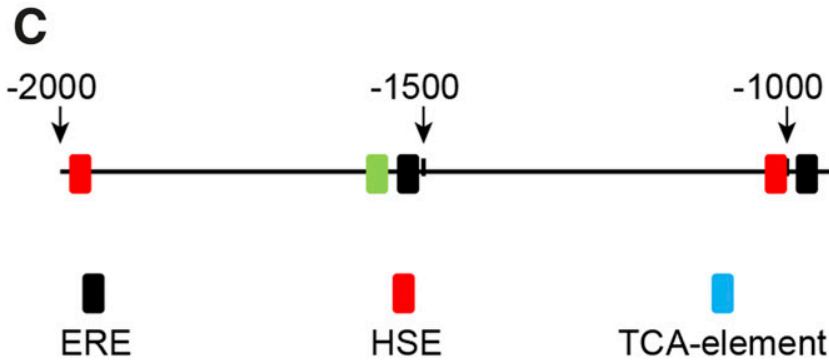

Fig. 1. Multiple comparison and cis-element analysis of $C a C B L 1$. A. Three putative conserved EF-hand domains were analyzed by searching SMART website Black boxes represent EF-hand domain. B, Comparison of deduced amino acid sequence of $C a C B L 1$ with those of its homologs in other plant species, including StCBL1 (Solanum tuberosum CBL1, XP_006362481.1), SlCBL1 (Solanum lycopersicum CBL1, XP_004244554.1), PtCBL1 (Populus trichocarpa CBL1, ABO43660.1), GrCBL1 (Gossypium raimondii CBL1, XP 012472107.1), PeCBL9 (Populus euphratica CBL9, NP 001291252.1), CsCBL1 (Citrus sinensis CBL1, XP_006477069.1), VvCBL1 (Vitis vinifera CBL1, NP_001267901.1), NtCBL1 (Nicotiana tabacum CBL1, XP_016451516.1), AtCBL1 (Arabidopsis thaliana CBL1, NP_567533.1), and OsCBL1 (Oryza sativa japonica group CBL1, XP_015614268.1) were analyzed using DNAMAN7 (Lynnon Biosoft). Green shading indicates 50 to $75 \%$ identity, red shading 75 to $100 \%$ identity, and black shading 100\% identity. The EF-hand domain sequence was marked by red line. C, A diagrammatic drawing of proCaCBL1. The main cis elements, including four ethylene-responsive element (ERE) (black boxes), three heat-shock element (HSE) (red boxes), one TCA element (blue boxes), and two W boxes (green boxes) were harbored in the promoter of $C a C B L 1$. Scale bar = $250 \mathrm{bp}$. 
A -2000 bp ${ }_{\text {W-box-2 }}^{-1000 \text { bp }}$ W-box-1 ${ }_{\overline{\mathrm{P} 2}}^{0 \text { bp }}$ ATG

B

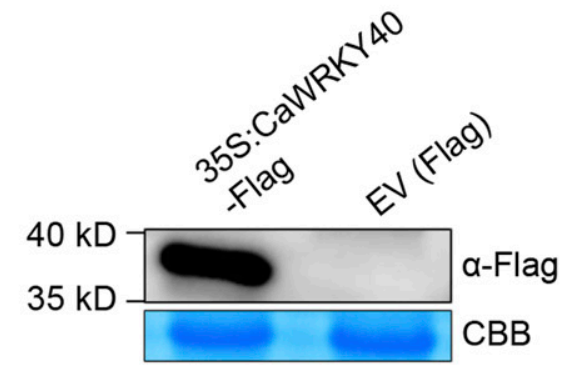

C

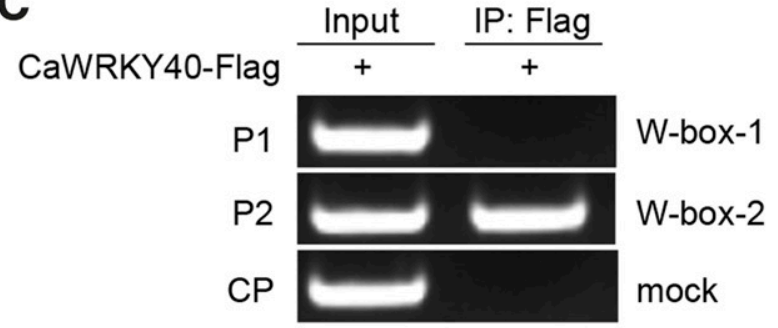

D

CaWRKY40 175 --GYQWRKYGQKVTRD-- 189

CaWRKY40-m 175 --GYQWRKYGMKVTRD-- 189

E W-box wild-type probe -1616--TTATAGTAAACTTAATATTAGTCAATTTTTAATCTAAAAATAGCAT-- -1571 W-box mutated probe -1616 --TTATAGTAAACTTAATATTAAAAAATTTTTAATCTAAAAATAGCAT-- 1571
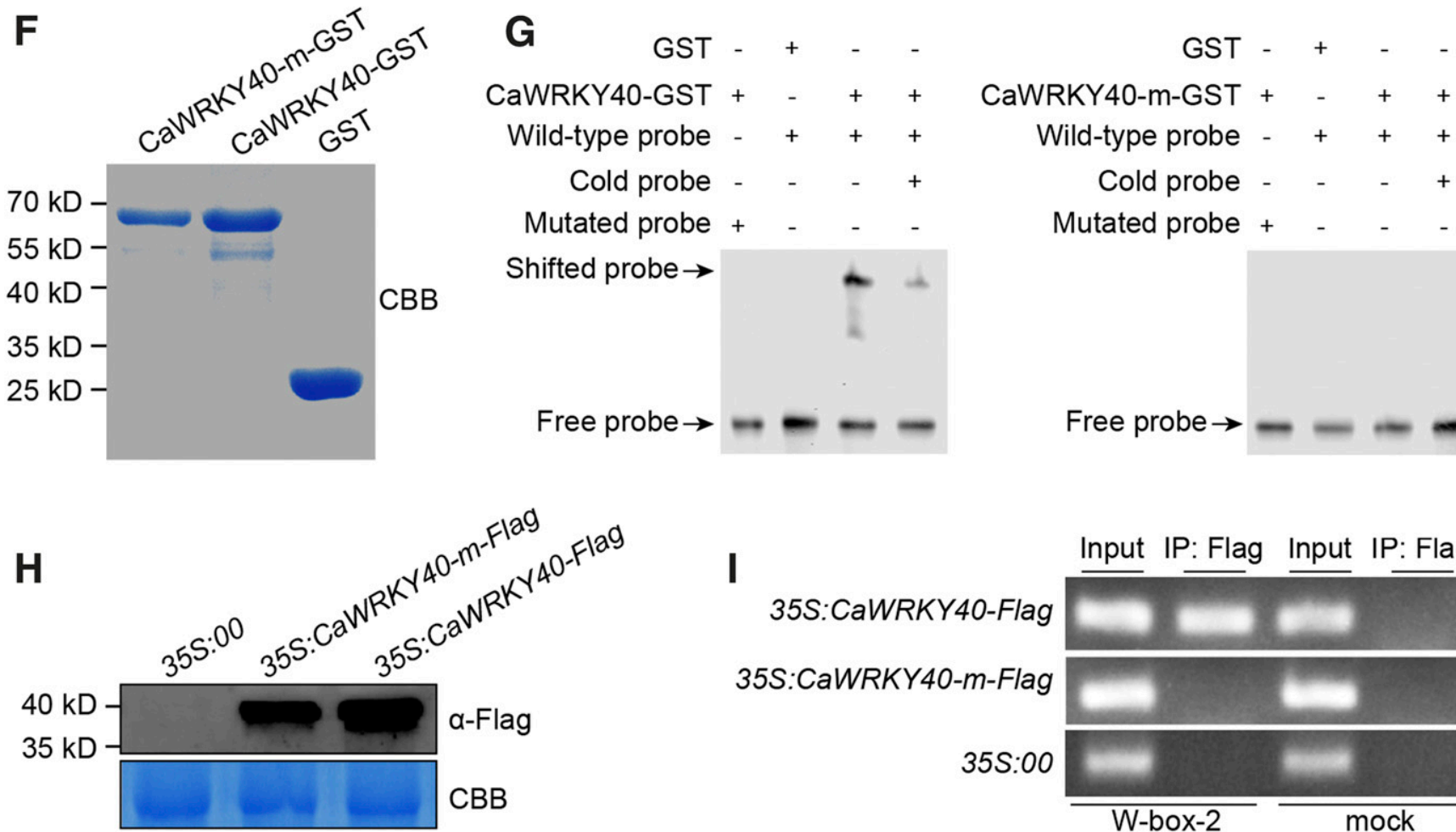

I

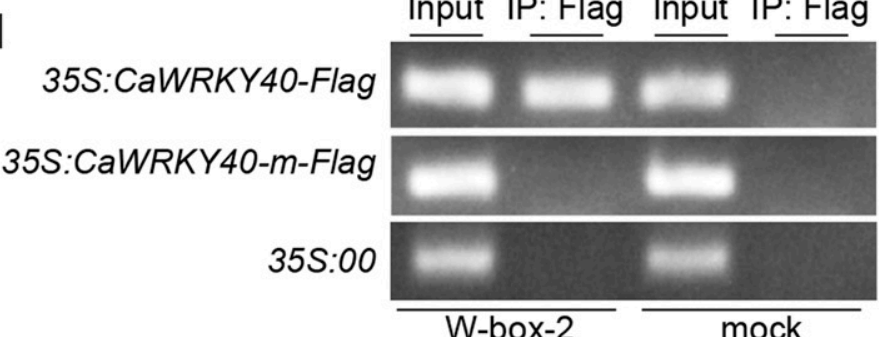

Fig. 2. The binding of CaWRKY40 to the promoter of $C a C B L 1$. A, The distribution of two W boxes in the promoter region of $C a C B L 1$ (proCaCBL1) and the corresponding two pairs of primers for chromatin immunoprecipitation PCR (ChIP-PCR). P1 = the primer pair based on the sequence flanking W-box-1 within proCaCBL1, $\mathrm{P} 2$ = the primer pair based on the sequence flanking W-box-2 within proCaCBL1, $\mathrm{CP}=$ the control primer pair based on a fragment (mock) without any $\mathrm{W}$ box within proCaCBL1 (as a negative control). W-box-1 = the first $\mathrm{W}$ box in proCaCBL1, W-box-2 = the second $\mathrm{W}$ box in $p r o C a C B L 1$. Scale bar $=250$ bp. B. The success of $C a C B L 1$ transient overexpression in pepper leaves were detected by Western blotting with anti-Flag antibody at $48 \mathrm{~h}$ postinfiltration. $\alpha$-Flag $=$ anti-Flag antibody, $\mathrm{CBB}=$ Coomassie brilliant blue, and EV = empty vector containing a Flag protein tag. C, CaWRKY40 bound to proCaCBL1 in vivo detected by ChIP-PCR. D, Deduced amino acid sequence of CaWRKY40 (wild-type) or CaWRKY40-m (mutated-type) from 175 to 189 containing core sequence of WRKY domain. E, The nucleotide sequence of wild-type or mutated-type probe of W-box-2 from -1,571 to -1,616. Red and blue letters indicate wild-type W-box-2 and mutated W-box-2, respectively. F, Purification of CaWRKY40-m-GST, CaWRKY40-GST, and glutathione-S-transferase (GST) protein. CBB = Coomassie brilliant blue. G, The binding of CaWRKY40-GST or CaWRKY40-m-GST to the W-box-2 cis element containing CaCBL1 promoter fragment by electrophoretic mobility shift assay in vitro. H, Immunoblot analysis of the transient overexpression of EV, CaWRKY4O-Flag, and CaWRKY40-m-Flag in pepper leaves. I, ChIP-PCR analysis of the binding of CaWRKY40 or CaWRKY40-m transiently overexpressed in pepper leaves to the W-box-2 containing a $C a C B L 1$ promoter fragment. 
(Fig. 2D) and the W-box-2 in $C a C B L 1$ promoter, used as a probe, was also mutated (Fig. 2E). In addition, CaWRKY40GST, CaWRKY40-m-GST recombinant protein, and glutathione-S-transferase (GST) protein (acting as a native control) expressed in Escherichia coli BL21 (DE3) were isolated and purified (Fig. 2F). The result of EMSA showed that the wild-type W-box-2 containing the promoter fragment of $C a C B L 1$ was bound by CaWRKY40-GST but was not bound by CaWRKY40m-GST, while the binding of CaWRKY40-GST to the probe containing the mutated W-box-2 was significantly decreased (Fig. 2G). This result indicate that CaWRKY40 binds to $C a C B L 1$ promoter via W-box-2. We further investigated the specific binding of CaWRKY40 to CaCBL1 promoter in vivo by ChIP-PCR assay. The pepper leaves infiltrated with Agrobacterium sp. strain GV3101 containing 35S:CaWRKY40-Flag, 35S:CaWRKY40-m-Flag, or 35S:00 were harvested for ChIP assay. The success of CaWRKY40-Flag and CaWRKY40-mFlag expression was detected by Western blot with anti-Flag antibody (Fig. 2H). The result of ChIP-PCR showed that the Wbox-2 containing $C a C B L 1$ promoter fragment was bound by CaWRKY40-Flag but was not bound by CaWRKY40-m-Flag in vivo (Fig. 2I). All these results suggest that CaWRKY40 directly bind to W-box-2 within $C a C B L 1$ promoter.

\section{The transcriptional regulation of the expression of CaCBL1 by CaWRKY40.}

As CaWRKY40 directly binds to W-box-2 within the $C a C B L 1$ promoter, we speculate that $C a C B L 1$ might be regulated by CaWRKY40 at the transcriptional level. We tested this hypothesis by assaying the effect of CaWRKY4O silencing on transcription of $C a C B L 1$. For CaWRKY4O silencing, a CaWRKY4O-specific 3' untranslated region fragment was used for virus-induced gene silencing (VIGS) vector construction. The efficiency of silencing was determined by assaying transcript levels of CaWRKY4O in a $\mathrm{MgCl}_{2}$ solution injection under room temperature (RT) $\left(\mathrm{MgCl}_{2} / \mathrm{RT}\right)-$, RSI under RT (RSI/RT)-, $\mathrm{MgCl}_{2}$ solution injection under $\mathrm{HT}\left(\mathrm{MgCl}_{2} / \mathrm{HT}\right)$-, or RSI under HT (RSI/HT)-challenged TRV:CaWRKY4O plants, using qRT-PCR. The result showed that the transcript level of CaWRKY4O in RSI/RT-, $\mathrm{MgCl}_{2} / \mathrm{HT}$-, or RSI/HT-challenged CaWRKY4O-silenced pepper plants was significantly lower than that in control plants (Fig. 3A), indicating the success of CaWRKY4O silencing in pepper plants by VIGS. The transcript level of $C a C B L 1$ in CaWRKY40-silenced pepper plants was significantly lower than that in the control plants (Fig. 3B). Similarly, the transcript level of $C a C B L 1$ was significantly lower in CaWRKY40-SRDX (chimeric dominant repressor version of CaWRKY4O) (Ishida et al. 2007; Ito et al. 2007) transiently overexpressing pepper plants than that in the control plants. By contrast, the transcript level of $C a C B L 1$ was significantly higher in CaWRKY4O transiently overexpressing pepper plants than that in the control plants (Fig. $3 \mathrm{C})$. All these data suggest that $C a C B L 1$ was positively regulated by CaWRKY40 in transcriptional level.

\section{CaCBL1 was upregulated transcriptionally by RSI/RT, $\mathrm{MgCl}_{2} / \mathrm{HT}$, and RSI/HT.}

As CaWRKY40 acts as a positive regulator in the pepper response to $R$. solanacearum infection and heat stress (Dang et al. 2013) and $C a C B L 1$ was positively regulated by CaWRKY40, we speculated that $C a C B L 1$ might be involved in immunity against RSI. To test this possibility, we first checked the response of $\mathrm{CaCBL} 1$ to RSI under RT $\left(30^{\circ} \mathrm{C}, 80 \%\right.$ humidity) and $\mathrm{HT}\left(37^{\circ} \mathrm{C}, 80 \%\right.$ humidity), by qRT-PCR. The result showed that $C a C B L 1$ was significantly upregulated by RSI under RT at $24 \mathrm{hpi}$ and by HT not until $48 \mathrm{~h}$ posttreatment (hpt). Noticeably, the upregulation of $C a C B L 1$ by RSI/RT was compromised by HT at $48 \mathrm{hpt}$ to a level that was still higher than that in the control plants (Fig. 4A), indicating that although the upregulation of $C a C B L 1$ by RSI is impaired by HT, its enhanced expression might also confer immunity. In parallel, the transcription of CaWRKY4O was also assayed. The results showed that CaWRKY4O was upregulated by RSI under HT individually and simultaneously at 24 and $48 \mathrm{hpt}$ (Fig. 4B). All these data indicate that $C a C B L 1$ and $C a W R K Y 4 O$ are activated by RSI under RT or HT, supporting the role of $C a C B L 1$ as positive regulator functionally and expressionally associated with CaWRKY4O during pepper response to RSI under RT or HT.

\section{The transcript level of $C a C B L 1$ is distinctly different in pepper lines with different $R$. solanacearum resistance levels.}

To assay the possible role of $C a C B L 1$ in pepper resistance to RSI under RT or HT, the transcript level of $C a C B L 1$ in three pepper lines, HN42 (a middle resistant variety to $R$. solanacearum under HT), SN (a highly resistant variety to $R$. solanacearum under HT), and TT5302 (a susceptible variety to $R$. solanacearum), were assayed. The result showed that plants of three lines exhibited no phenotypic difference against $\mathrm{HT}\left(37^{\circ} \mathrm{C}, 80 \%\right.$ humidity) as well as RT but exhibited distinct levels of disease resistance and transcript levels of $C a C B L 1$ in
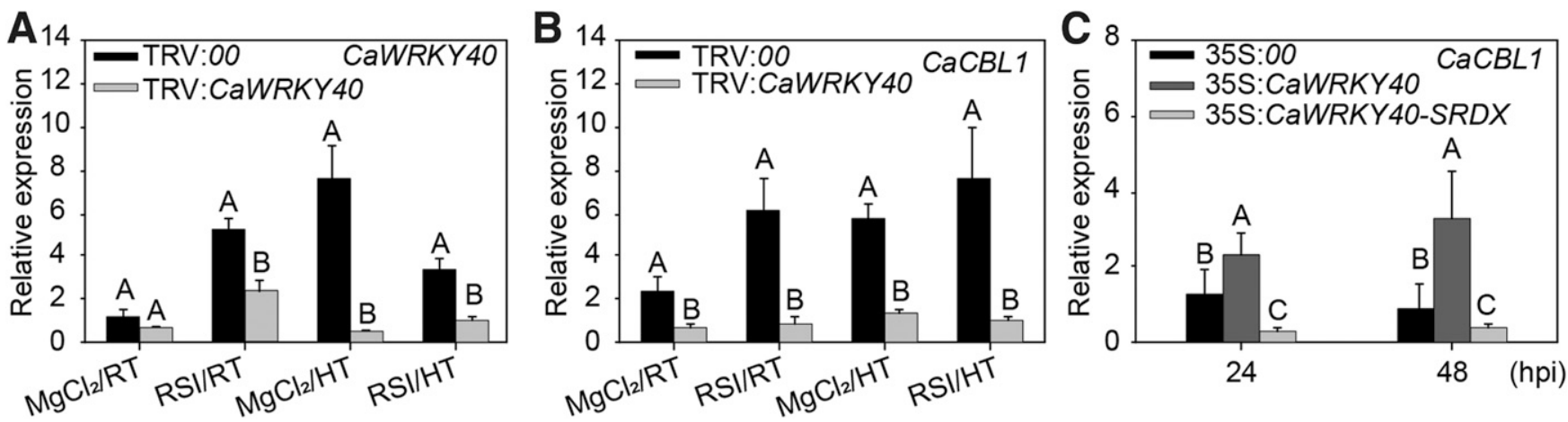

Fig. 3. Relative transcript level of CaCBL1 was analyzed by quantitative real-time PCR in CaWRKY40-silenced and 35S:CaWRKY4O-Flag or 35S:CaWRKY40SRDX-Flag transiently overexpressed pepper leaves. A, The success of CaWRKY40 silencing by virus-induced gene silencing in pepper plants challenged with $\mathrm{MgCl}_{2}$ at room temperature $\left(\mathrm{MgCl}_{2} / \mathrm{RT}\right)$, Ralstonia solanacearum inoculation ( $\left.\mathrm{RSI}\right)$ at room temperature $(\mathrm{RSI} / \mathrm{RT}), \mathrm{MgCl}$ at high temperature $\left(\mathrm{MgCl}_{2} / \mathrm{HT}\right)$, or $\mathrm{RSI} / \mathrm{HT}$. B, The transcript level of $C a C B L 1$ was downregulated by silencing of $C a W R K Y 40$ in pepper plants with or without the challenge of $\mathrm{MgCl} / \mathrm{RT}_{2}$, $\mathrm{RSI} / \mathrm{RT}, \mathrm{MgCl}_{2} / \mathrm{HT}$, or RSI/HT. C, The transcript level was enhanced by transient overexpression of $C a W R K Y 40$ while decreased by that of $C a W R K Y 40-S R D X$. The relative transcript levels of the tested genes were compared with those in the mock-treated control plants, which were set to a relative expression level of 1 , the values were the means \pm standard deviation from four biological replications, with one plant per biological replicate. Statistical analyses were performed using Fisher's protected least significant difference test. Different upper-case letters indicate significant difference $(P<0.01)$ 
the three $R$. solanacearum-inoculated lines under HT. The line $\mathrm{SN}$, with the highest disease resistance under RSI/HT, displayed the highest transcript level of CaCBL1; the line TT5302, exhibiting the most serious disease under RSI/HT, displayed the lowest transcript level of $C a C B L 1$ (Fig. 5A and B). These data indicate that transcript levels of $C a C B L 1$ might be positively related to disease resistance level under RSI/HT in different pepper lines.

\section{The subcellular localization of CaCBL1.}

To assay its possible subcellular localization, we predicted the CaCBL1 subcellular localization, using the PSORT online tool. The result showed that CaCBL1 may locate in the cytoplasm $(39.1 \%)$ and nuclei $(17.4 \%)$. To verify this possibility,

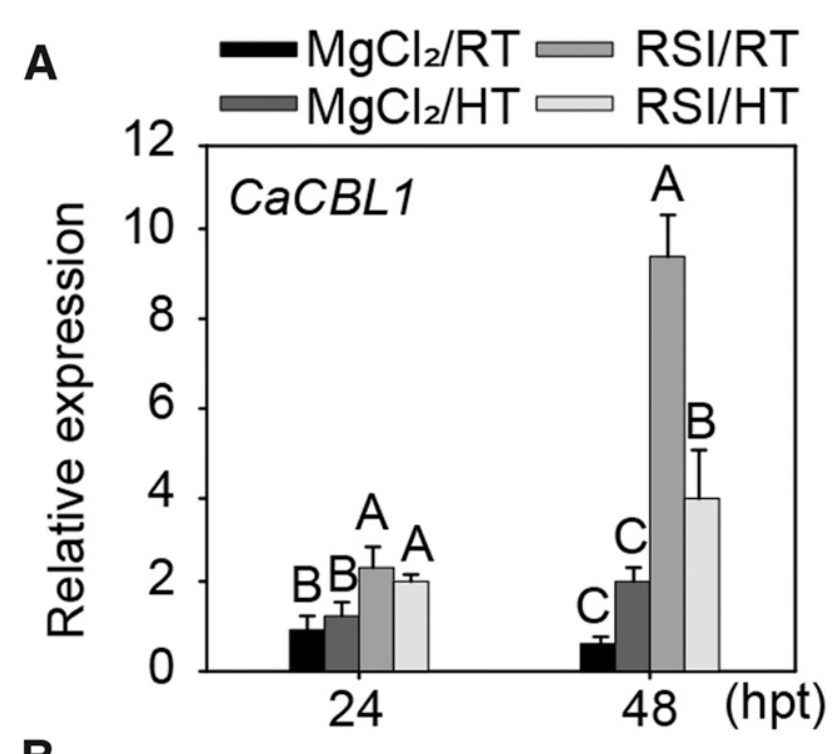

B

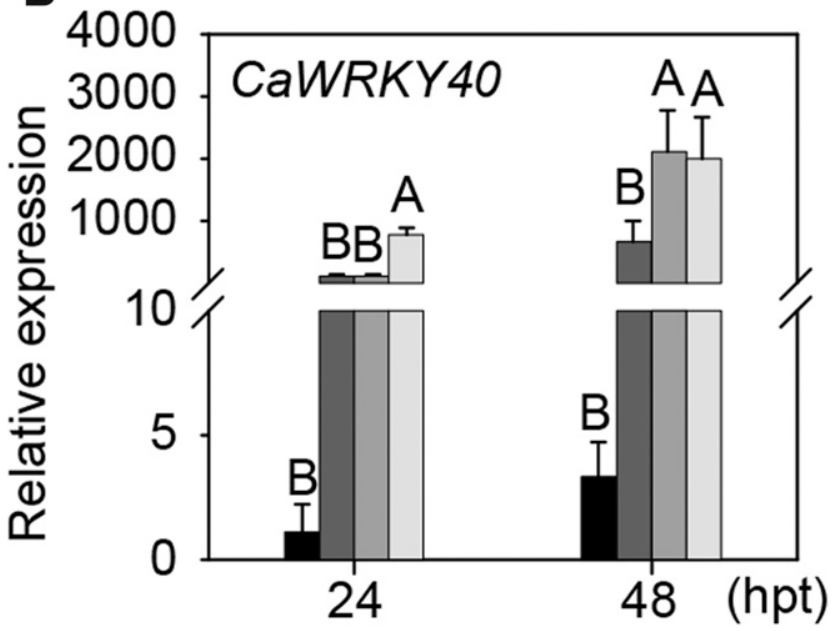

Fig. 4. Response of $C a C B L 1$ and $C a W R K Y 40$ to $\mathrm{MgCl}_{2}$ at room temperature $\left(\mathrm{MgCl}_{2} / \mathrm{RT}\right)$, Ralstonia solanacearum inoculation (RSI) at room temperature (RSI/RT), $\mathrm{MgCl}_{2}$ at high temperature $\left(\mathrm{MgCl}_{2} / \mathrm{HT}\right)$, or RSI at high temperature (RSI/HT). A and $\mathbf{B}$, Pepper plants of GZ03 at the six- to eightleaf stage with their roots damaged were challenged with $\mathrm{MgCl}_{2} / \mathrm{RT}$, $\mathrm{RSI} / \mathrm{RT}, \mathrm{MgCl}_{2} / \mathrm{HT}$, or RSI/HT, and their roots were harvested and frozen by liquid nitrogen at 24 and $48 \mathrm{hpt}$ to isolate the total RNA for measuring the relative transcript level of CaCBL1 and CaWRKY4O by quantitative real-time PCR. The relative transcript levels of the tested genes were compared with those in the mock-treated control plants, which were set to a relative expression level of "1", the values are the means \pm standard deviation from four biological replications. Statistical analyses were performed using Fisher's protected least significant difference test. Different upper-case letters indicate significant difference $(P<0.01)$. the subcellular localization assay was further performed by transient overexpression of CaCBL1-GFP fusion protein in Nicotiana benthamiana leaves, and the subcellular localization of CaCBL1 was detected by observing green fluorescent protein (GFP) signals. We generated 35S:CaCBL1-GFP construct. The fused CaCBL1-GFP protein was expressed in N. benthamiana leaves by infiltration of Agrobacterium tumefaciens GV3101 containing 35S:CaCBL1-GFP, the GFP signals in epidermal cells was detected at 48 hpi using a confocal microscope. GFP signals within 35S:GFP-expressed epidermal cells of $N$. benthamiana leaves were observed in the cytoplasm and nuclei. Similarly, the GFP signals of CaCBL1-GFP expressed cells were also visualized in the plasma membrane, cytoplasm, and the nuclei (Fig. 6A). To further confirm this result, the cytoplasmic and nuclear proteins

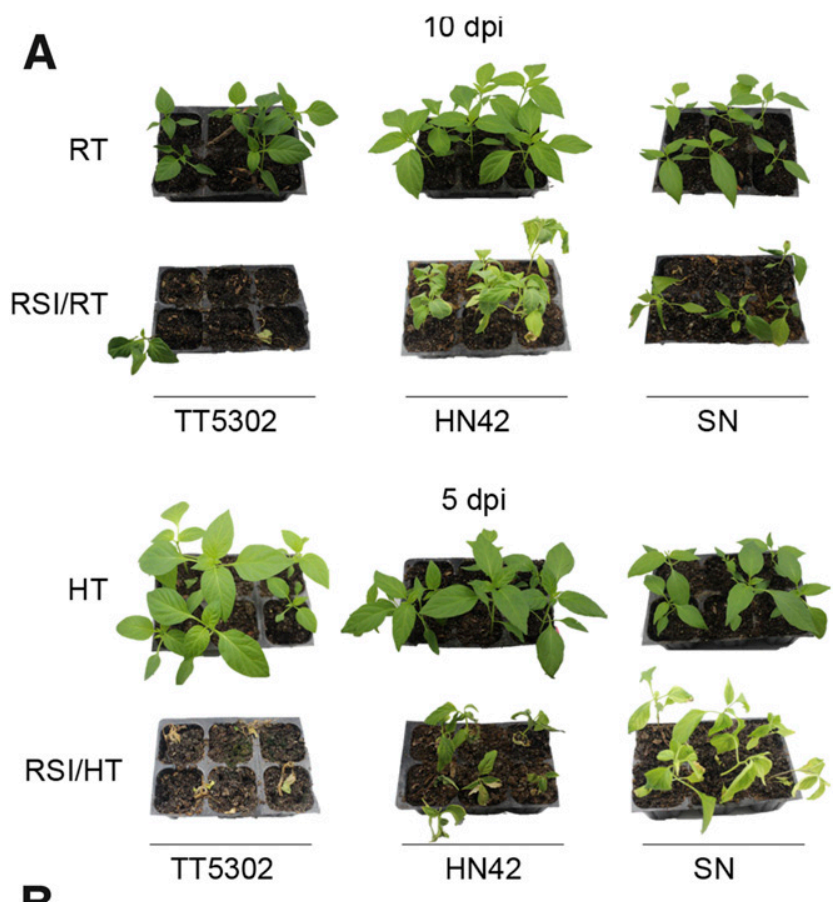

B

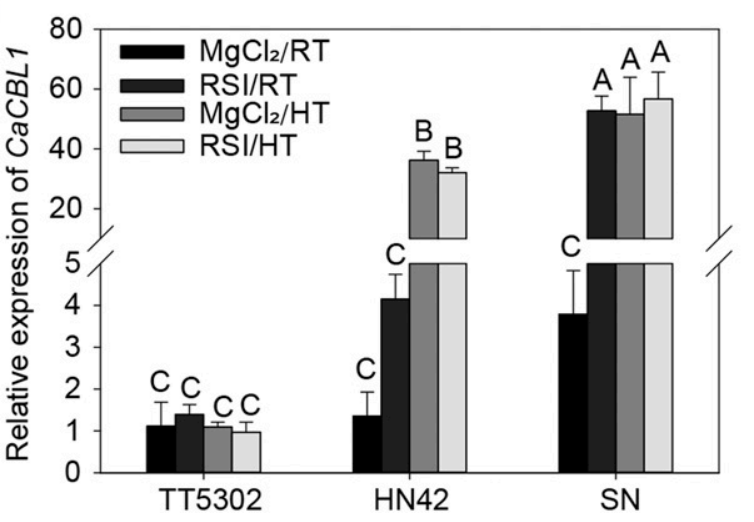

Fig. 5. The phenotype and $C a C B L 1$ transcript level in three pepper lines. A The phenotype of pepper resistance to room temperature (RT) (acted as a control) or R. solanacearum inoculation (RSI) under RT at 10 dpi and to HT (acted as a control for RSI under high temperature [HT] [RSI/HT]) or RSI under HT at 5 dpi in three pepper lines TT5302, HN42, and SN. B, The relative transcript level of $C a C B L 1$ was analyzed by quantitative real-time PCR in three pepper lines roots with $\mathrm{MgCl}_{2}$ at room temperature $\left(\mathrm{MgCl}_{2} / \mathrm{RT}\right)$, Ralstonia solanacearum inoculation (RSI) at room temperature (RSI/RT), $\mathrm{MgCl}_{2}$ at high temperature $\left(\mathrm{MgCl}_{2} / \mathrm{HT}\right)$, or RSI/HT at $48 \mathrm{~h}$ posttreatment. The transcript level of $C a C B L 1$ was compared with those in the RT control plants, which were set to a relative expression level of 1 , the values were the means \pm standard deviation from four biological replications. Statistical analyses were performed using Fisher's protected least significant difference test. Different upper-case letters indicate significant difference $(P<0.01)$. 
were isolated from $C a C B L 1-G F P$ or $G F P$ transiently overexpressing $N$. benthamiana leaves, respectively, which were further subjected to Western blot assay against anti-GFP antibody. The result consistently showed that GFP signal presented in both the cytoplasmic and nuclear proteins (Fig. 6B), indicating that CaCBL1 locate to the whole cells, including plasma membrane, cytoplasm, and the nuclei, in which the nuclei and plasma membrane were displayed by immunoblotting with antibody of histone 3 (H3) (Choi et al. 2012) and heat-shock complex 70 (Choi et al. 2012), respectively (Fig. 6B).

As the transcript levels of $C a C B L 1$ are different in pepper lines with different $R$. solanacearum resistance levels, we further investigated the subcellular localization of CaCBL1 in pepper lines leaves of TT5302 and HN42 under conditions at 30 or $37^{\circ} \mathrm{C}$. The results showed that CaCBL1 locate to the whole cells including plasma membrane, cytoplasm, and the nuclei in leaf epidermic cells of both TT5302 and HN42 pepper, indicating that subcellular localization of CaCBL1 is conserved across pepper lines with different levels of disease resistance and is not affected by temperature (Supplementary Fig. S2).

\section{Silencing of $C a C B L 1$ enhances susceptibility of pepper to $R$. solanacearum.}

To assay the role of $C a C B L 1$ in the GZ03 (a middle resistant variety to $R$. solanacearum under HT) pepper plant response to $R$. solanacearum, we used VIGS to generate $C a C B L 1$-silenced pepper seedlings. The relative expression level of $C a C B L 1$ in TRV:CaCBL1 pepper seedlings were significantly reduced to approximately $4 \%$ of those in the TRV:00 plants (Fig. 7A), and silencing of $C a C B L 1$ by VIGS appeared to be specific, since the relative transcription expression level of $C a C B L 3$, which exhibits the highest sequence similarity to $C a C B L 1$ among all of the CBL genes in pepper genome, was unaffected by the silencing of $C a C B L 1$, and transcript levels of $C a C B L 3$ in $R$. solanacearum-inoculated TRV:CaCBL1 pepper plants under RT $\left(30^{\circ} \mathrm{C}, 80 \%\right.$ humidity) or HT $\left(37^{\circ} \mathrm{C}, 80 \%\right.$ humidity) were significantly higher than that in the TRV:00 control plants, only in HT-treated TRV:CaCBLI pepper plants was the relative transcript level of $C a C B L 3$ lower than that in the control plants (Supplementary Fig. S3).

The TRV:CaCBL1 pepper plants were used to assay the effect of $C a C B L 1$ silencing on pepper resistance to RSI under RT or HT conditions. The result showed that HT accelerated the development of disease in both $R$.solanacearum-inoculated TRV:00 and TRV:CaCBLI plants. While the TRV:CaCBLI plants exhibited the quickest and most severe wilting symptoms as well as the highest disease indices at all the tested timepoints among $R$. solanacearum-inoculated TRV:00 and TRV:CaCBL1 plants. While the TRV:CaCBL1 plants exhibited the quickest and most severe wilting symptoms as well as the highest disease indices at all the treated timepoints among $R$. solanacearum inoculated TRV:00 and TRV:CaCBL1 plants under RT and HT (Fig. 7B and $\mathrm{C})$. In addition, $R$. solanacearum growth in $C a C B L 1$-silenced pepper leaves significantly increased compared with the control pepper leaves under both RT and HT (Fig. 7D).

To confirm the phenotypic effect of $C a C B L 1$ silencing, the transcript levels of pepper immunity-associated marker genes including CaNPR1, CaDEF1 (Do et al. 2004), CaCDPK15 (Shen et al. 2016b), CabZIP63 (Shen et al. 2016a), and CaWRKY40 (Dang et al. 2013) were measured in TRV:CaCBL1 and TRV:00 pepper plants under the challenges of $\mathrm{MgCl}_{2} / \mathrm{RT}$, $\mathrm{RSI} / \mathrm{RT}, \mathrm{MgCl}_{2} / \mathrm{HT}$, or RSI/HT. All of the tested genes exhibited no altered transcription between the mock-treated TRV:CaCBL1 and TRV:00 pepper plants. When challenged by HT, only $C a D E F 1$ and $C a C D P K 15$ exhibited significantly lower transcript levels in TRV:CaCBL1 plants compared with that in TRV:00 plants, the other tested marker genes did not alter their expression in TRV:CaCBL1 plants compared with TRV:00 plants. Upon challenge by $R$. solanacearum under RT, all of the tested marker genes exhibited decreased transcript levels in TRV: CaCBL1 plants compared with TRV:OO plants. It is noticeable that the relative transcript levels of the tested marker genes were the lowest in RSI/HT-challenged TRV:CaCBL1 (Fig. 7E). These results indicate that $\mathrm{CaCBL} 1$ acts as a positive regulator in pepper response to $R$. solanacearum attack under both RT and HT and the downregulation of the tested marker genes might contribute to the enhanced susceptibility of TRV:CaCBL1 plants to RSI under RT or HT.

\section{Transient overexpression of $C a C B L 1$ induces expression} of cell death and defense-related genes in pepper plants.

The role of CaCBL1 as a positive regulator in pepper response to RSI was further assayed by assaying the effect of its transient overexpression on hypersensitive response (HR) mimic cell death as well as the expression of immunityassociated marker genes in pepper leaves. The pepper leaves of GZ03 were infiltrated with Agrobacterium sp. strain GV3101 harboring 35S:CaCBL1-Flag or 35S:Flag constructs. By Western blot with the protein isolated from CaCBL1-Flag transiently overexpressing pepper leaves and anti-Flag antibody, CaCBL1-Flag appeared to be successfully expressed in pepper leaves (Fig. 8A). Transient overexpression of $\mathrm{CaCBL1-}$ Flag triggered obvious HR mimic cell-death response at 4 days postinfiltration (Fig. 8B). The cell death was assessed by trypan blue staining, no or only very weak trypan blue staining was visualized in leaves infiltrated with Agrobacterium sp. strain GV3101 containing the empty vector, whereas transient overexpression of 35S:CaCBL1-Flag produced darker trypan blue staining in pepper leaves. Since hydrogen peroxide $\left(\mathrm{H}_{2} \mathrm{O}_{2}\right)$ is

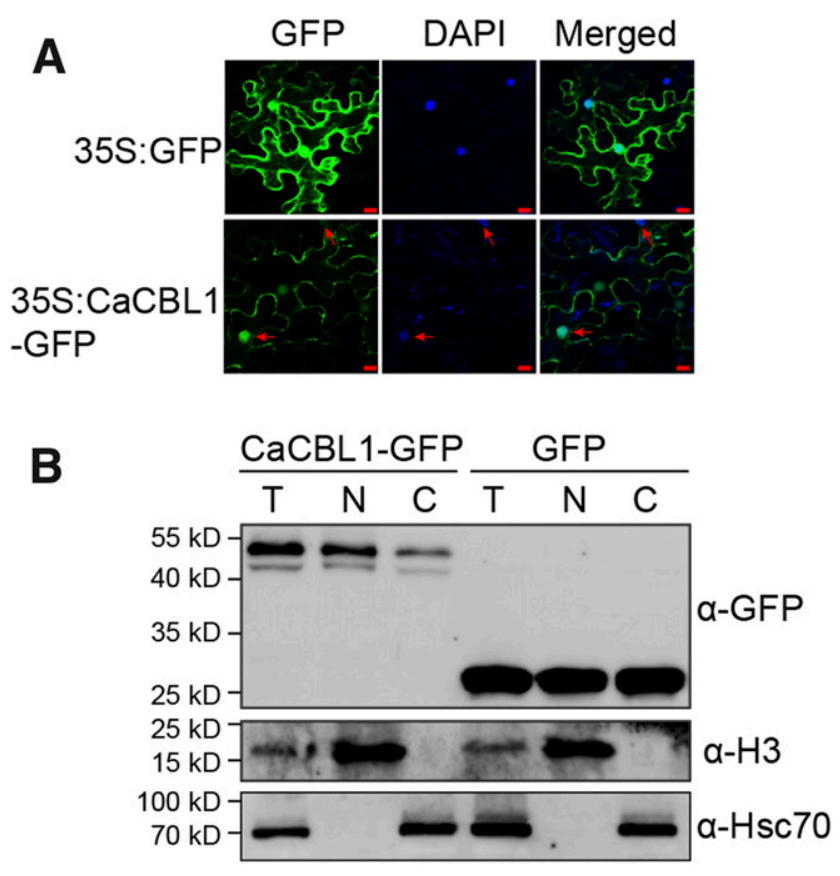

Fig. 6. Subcellular localization of CaCBL1. A, Subcellular localization of green fluorescence protein (GFP) (as a control) or CaCBL1-GFP protein by transient overexpression of $35 S: G F P$ or the $35 S: C a C B L 1-G F P$ construct in Nicotiana benthamiana leaves. Bars $=50 \mu \mathrm{m}$. The images were taken by Leica confocal microscopy at $48 \mathrm{~h}$ postinfiltration (hpi). B, Protein gel blot analysis of total $(\mathrm{T})$, cytosolic $(\mathrm{C})$, and nuclear $(\mathrm{N})$ protein extracts from 35S:GFP and 35S:CaCBL1-GFP transiently overexpressing N. benthamiana leaves at 48 hpi by Western blotting with anti-GFP antibody. Histone $\mathrm{H} 3$ and Hsc70 were used as markers targeting the nucleus and cytoplasm, respectively. $\alpha$-GFP $=$ anti-GFP antibody, $\alpha-\mathrm{H} 3=$ antihistone $\mathrm{H} 3$ antibody, and $\alpha-\mathrm{Hsc} 70=$ anti-Hsc70 antibody. 
closely related to HR, production in CaCBLI-Flag transiently overexpressing pepper leaves was measured by diaminobenzidine (DAB) staining. The result showed that there was visible DAB staining in the 35S:CaCBL1-Flag transiently expressed leaves but not in the leaves infiltrated with Agrobacterium sp. strain GV3101 containing the empty vector (Fig.
$8 C)$. Ion leakage was also measured to analyze the severity of cell death in 35S:CaCBL1-Flag transiently overexpressed pepper leaves. The result showed that higher levels of ion leakage were found at 24 and $48 \mathrm{hpi}$ in $C a C B L 1$ overexpressing pepper leaves than that in the pepper leaves infiltrated with Agrobacterium sp. strain GV3101 containing empty vector (Fig. 8D).
A

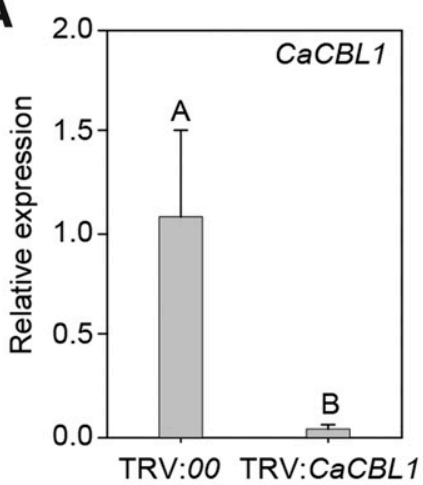

C
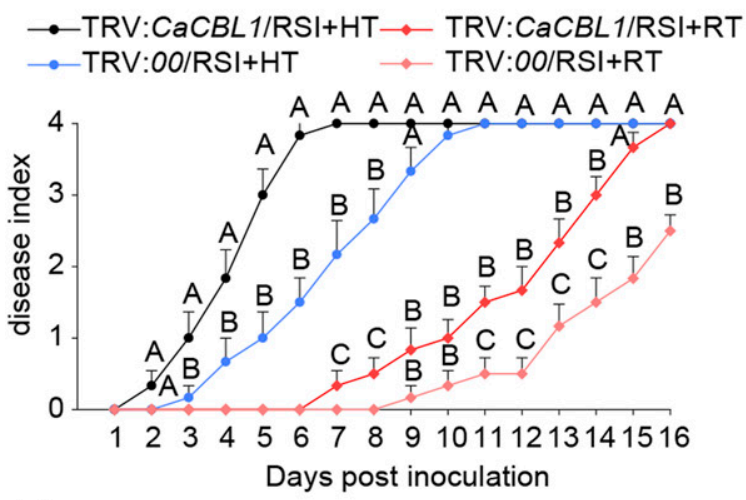

D

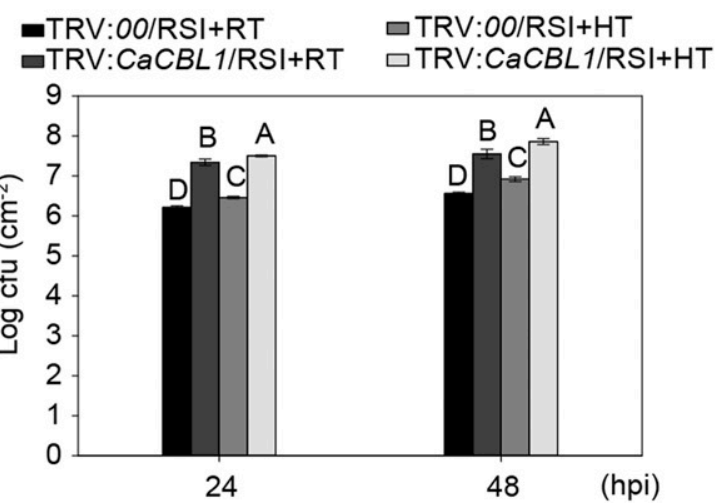

B

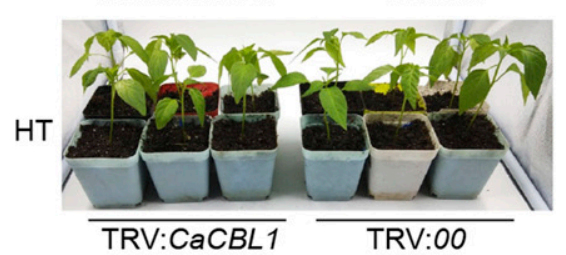

E
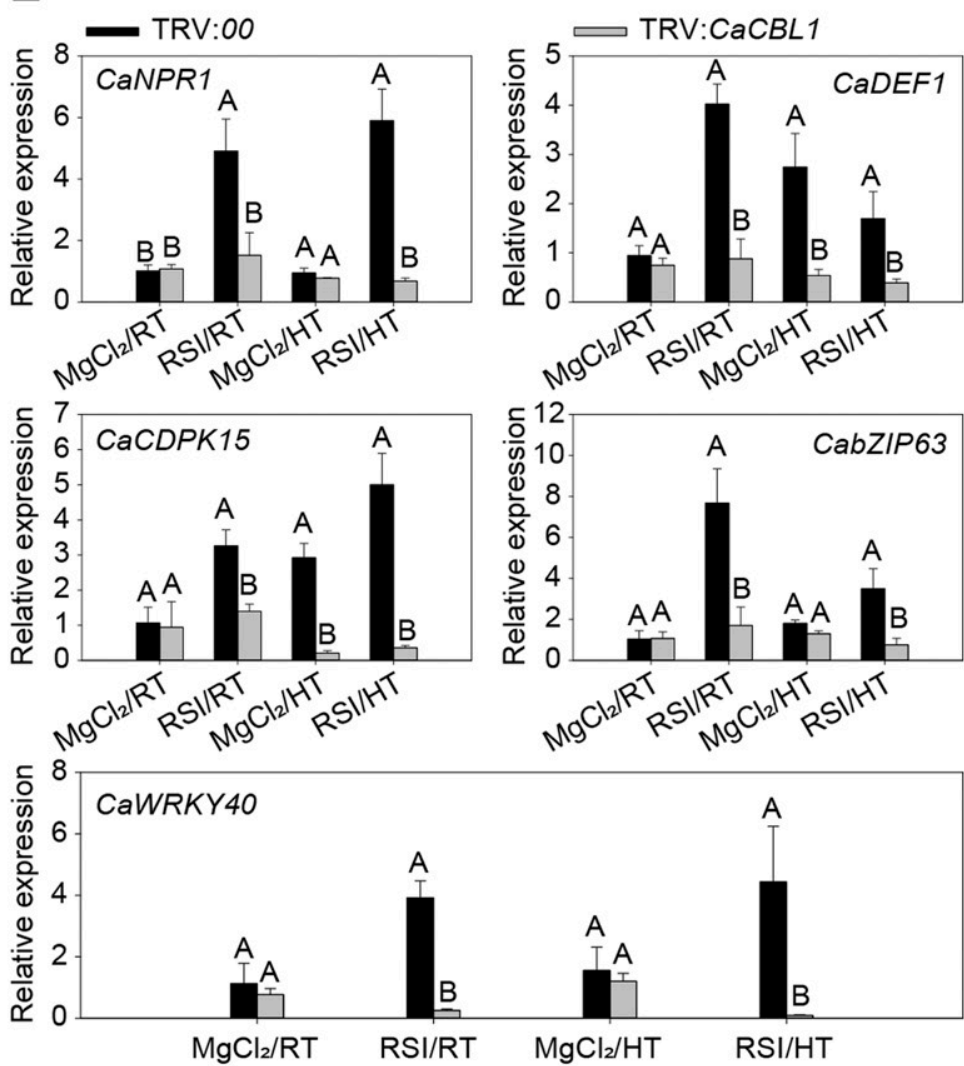

Fig. 7. The effect of $C a C B L 1$-silencing on pepper response to Ralstonia solanacearum inoculation (RSI) at $30^{\circ} \mathrm{C}$ with $80 \%$ humidity (RSI/RT) and to RSI at $37^{\circ} \mathrm{C}$ with $80 \%$ humidity (RSI/HT). A, The relative transcript level of $C a C B L 1$ in the leaves of TRV:00 or TRV:CaCBL1 pepper plants by quantitative real-time PCR (qRT-PCR). B, Symptoms of RSI TRV:CaCBL1 pepper plants under HT conditions at 5 days postinoculation (dpi) (left) and under RT at 13 dpi (right), respectively. TRV:00 pepper plants were used as control. Pepper plants treated with only HT or RT were considered to be control pepper plants compared with those treated with RSI/HT or RSI/RT. C, Disease index of RSI TRV:CaCBL1 pepper plants under RT or under HT from 1 to 16 dpi. The disease index (mean \pm standard deviation) was determined according to the percentage of the wilted leaves on 24 inoculated pepper plants. The values are defined as $0=$ no symptoms, $1=0$ to $25 \%$ wilted leaves, $2=25$ to $50 \%, 3=50$ to $75 \%, 4=75$ to $100 \%$ (Mukhtar et al. 2008). TRV:00/RSI or TRV:00/RSI/HT means the TRV:00 pepper plants were treated with RSI under RT or RSI under HT, and TRV:CaCBL1/RSI/RT or TRV:CaCBL1/RSI/HT means the TRV:CaCBL1-silenced pepper plants were also treated with RSI under RT or with RSI under HT. D, Detection of $R$. solanacearum growth displayed as colony-forming units (CFU) in leaves of silenced-CaCBL1 and control pepper plants under the condition of RT or HT treatment. E, The transcript levels of the defense-related genes including CaWRKY40, CaNPR1, CaDEF1, CaCDPK15, and CabZIP63 in TRV:00 or TRV:CaCBL1 pepper plant roots challenged with RSI/RT, HT, or RSI/HT by qRT$\mathrm{PCR}$ analysis at $48 \mathrm{~h}$ posttreatment. In $\mathrm{A}$ and $\mathrm{D}$, the transcript levels of the tested genes were compared with those in the mock-treated (injected $10 \mathrm{mM} \mathrm{MgCl}_{2}$ solution into pepper leaves) control plants, which were set to a relative expression level of 1 . Values are the means \pm standard deviation from four biological replications, with one plant per biological replicate. Statistical analyses were performed using Fisher's protected least significant difference test, different upper-case letters indicate significant difference $(P<0.01)$. 
In parallel, we examined transcript abundances of defenserelated genes including CaNPR1, CaWRKY40, CabZIP63, and CaCDPK15 (Fig. 8E). The result showed that the relative transcription levels of the tested defense-related genes were increased in $C a C B L 1$ transiently overexpressing pepper plant leaves compared with that in the control pepper plants. These data indicate that $C a C B L 1$ acts as a positive regulator in pepper response to RSI.

\section{DISCUSSION}

Bacterial wilt caused by $R$. solanacearum is one of the most important diseases on pepper, and this disease tends to be more serious under HT conditions. A better understanding of the molecular mechanism underlying pepper immunity toward $R$. solanacearum might benefit genetic improvement of pepper resistance to bacterial wilt. We provided evidence that CaCBL1 acts as a positive regulator in pepper resistance to $R$. solanacearum infection under RT and HT, forming a positive feedback loop with CaWRKY40.

Calcium plays a crucial role as a second messenger in mediating various defense responses under environmental stresses. CBLs, encoded by a small gene family in different plant species, have been implicated as important $\mathrm{Ca}^{2+}$ sensors via their EF-hand in calcium signaling (Mähs et al. 2013). However, information on the role of members of the CBL family in plant defense is very limited. Data in the present study showed that transcript abundance of $C a C B L 1$ was upregulated by RSI and that $C a C B L 1$ silencing by VIGS significantly enhanced susceptibility of pepper plants to RSI at RT or under HT. By contrast, the transient overexpression of $C a C B L 1$ triggered significantly HR mimic cell death, manifested by darker trypan blue staining and DAB staining. Based on these data, it can be concluded that $\mathrm{CaCBL} 1$ acts as a positive regulator in pepper immunity against $R$. solanacearum under HT. This result was supported by the data that SA-dependent CaNPRI and JAdependent $C a D E F 1$, which positively contribute to plant immunity (Do et al. 2004), were downregulated by CaCBL1 silencing while enhanced by its transient overexpression. In particular, CaWRKY40, CabZIP63, and CaCDPK15, the three positive regulators during pepper response to RSI (Dang et al. 2013; Shen et al. 2016a and b), were activated by CaCBL1 transient overexpression but downregulated by its silencing. These results support the role of CaCBL1 as a positive regulator in pepper response to RSI and indicate that this role is closely related to the signaling networks mediated by CaWRKY40, CabZIP63, and CaCDPK15. As CaWRKY40 is directly regulated by CabZIP63 (Dang et al. 2013; Shen et al. 2016a), a pathway can be speculated that, upon the perception of pathogen and initiation of $\mathrm{Ca}^{2+}$ influx, $C a C B L 1$ might be upregulated, which in turn activates the transcriptions of CabZIP63 and CaWRKY40, leading to massive defense transcriptional reprogramming. It is still unknown how CaCBL1 is associated with the TFs in the nucleus. One possibility is that interacting with some unidentified CIPKs (Luan 2009) might modulate some specific TFs or cofactors (Liu et al. 2019; Sanyal et al. 2017), e.g., CabZIP63 by phosphorylation, as CaCBL1 was found to be localized to the whole cells, consistent with the results of previous studies that CBLs and CIPKs localized the whole cell, including plasma

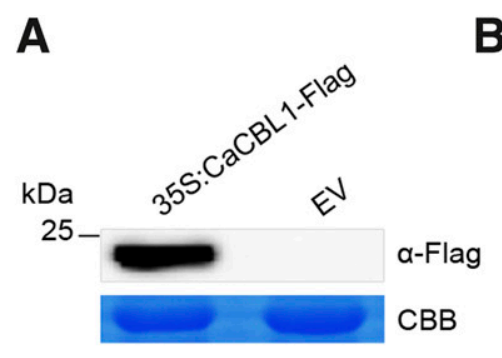

E

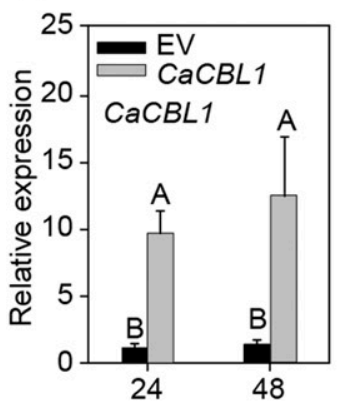

Hours post infiltration

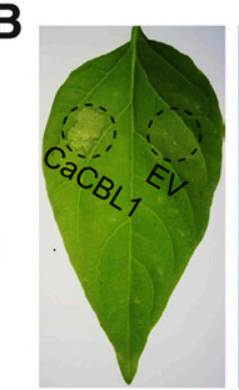

bright field

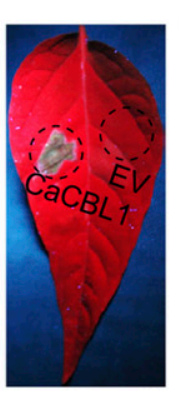

UV

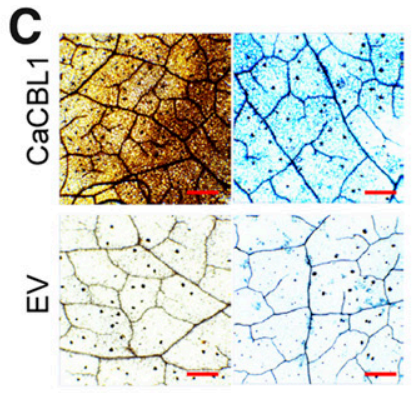

$\mathrm{DAB}$
Trypan blue

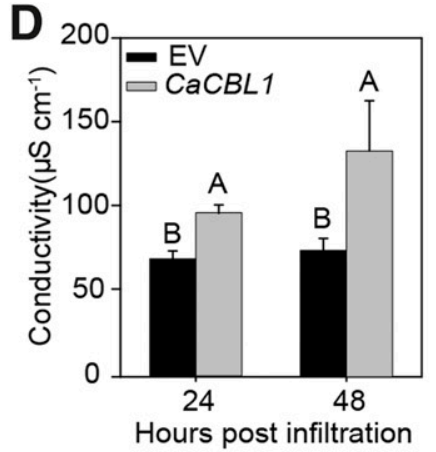

Fig. 8. The effect of transient overexpression of $C a C B L 1$ on the hypersensitive response (HR) mimic cell death and the expression of immunity-associated genes in pepper leaves. A, The success of 35S:CaCBL1-Flag transient overexpression in pepper leaves was detected by Western blotting of total proteins against antibody of Flag at $48 \mathrm{~h}$ postinfiltration (hpi). $\alpha$-Flag $=$ anti-Flag antibody and CBB $=$ Coomassie brilliant blue. B, Transient overexpression of CaCBL1 triggered significant cell death at 4 days postinfiltration, observed under bright field (left) and under UV light (right). EV = empty vector containing a Flag protein tag. $\mathbf{C}, \mathrm{H}_{2} \mathrm{O}_{2}$ accumulation and $\mathrm{HR}$ mimic cell death triggered by transient overexpression were manifested by diaminobenzidine staining (left) and trypan blue staining (right) at $3 \mathrm{dpi}$. Bars $=10 \mu \mathrm{m}$. D, Quantification of electrolyte leakage by ion conductivity to assess the cell-death response in pepper leaves. E, Relative transcript level of CaCBL1 and defense-related response genes, including CaNPR1, CaWRKY4O, CaCDPK15, and CabZIP63, in pepper leaves at 48 hpi, by quantitative real-time PCR. The transcript levels of the tested genes were compared with those in the mock-treated control plants, which were set to a relative expression level of 1 , the values are the means \pm standard deviation from four biological replications. Statistical analyses were performed using Fisher's protected least significant difference test, different lower- and upper-case letters indicate significant difference at $P<0.05$ and $P<0.01$, respectively. 
membrane, cytoplasm, and the nucleus (Batistič et al. 2010; Wang et al. 2016; Zhao et al. 2009). To confirm this hypothesis, further investigation is required.

CaCBL1 was also upregulated by HT and probably acts as a positive regulator in pepper thermotolerance, since CaWRKY40, a positive regulator in pepper thermotolerance (Dang et al. 2013), was positively regulated by CaCBL1. Thus, it can be speculated that CaCBL1 might act as a point of convergence for pepper response to RSI and HT. In addition, our data also indicate that $\mathrm{CaCBL} 1$ acts as a positive regulator in pepper immunity against RSI under HT. However, RSI plus HT did not induce additively the transcription of $C a C B L 1$ as expected. Instead, the transcript level of CaCBLI in $R$. solanacearuminoculated pepper plants under HT was significantly lower than that in $R$. solanacearum-inoculated pepper plants under RT, indicating that pepper response to simultaneously occurring RSI and HT is different from that to individual stresses. Similarly, previous studies demonstrated that unique transcriptional reprogramming was activated by different stress combinations compared with that by individual stresses (Kissoudis et al. 2016; Pandey et al. 2015; Zandalinas et al. 2020; Zhang et al. 2017). Our data indicate that the response of $C a C B L 1$ to RSI and to HT may interact and antagonize one another and this crosstalk might reflect a close relationship between growth of soil-borne pathogens of pepper and the occurrence of HT. A more serious damage caused by HT might be attributed to its combinational effect on pathogen infection, which might attenuate plant immunity mediated by resistance (R) proteins (Jambunathan et al. 2001; Wang et al. 2005, 2009; Xiao et al. 2003; Yoshioka et al. 2001; Zhang et al. 2017) while promoting pathogen growth (Fujita et al. 2006). Indeed, we found that $R$. solanacearum exhibited a high speed of growth at $37^{\circ} \mathrm{C}$ (Supplementary Fig. S4), and $R$. solanacearum-inoculated pepper plants exhibited accelerated pathogenesis under HT conditions $\left(37^{\circ} \mathrm{C}, 80 \%\right.$ humidity) than normal condition $\left(30^{\circ} \mathrm{C}\right.$, $80 \%$ humidity). The serious diseases caused by HT might exert continuous selective pressure upon pepper during its evolution, which might lead to some countermeasures in pepper. To maintain higher levels of CaCBL1 might be one of these countermeasures, which might be attributed to the evolution of HTinsensitive $\mathrm{R}$ proteins. Further identifying these $\mathrm{R}$ proteins would provide new insight into the mechanism underlying pepper immunity under HT. It is worth pointing out that, although it has been frequently found that a single gene encoding $\mathrm{Ca}^{2+}$ sensors such as CDPKs (Asano et al. 2012; Shen et al. 2016b), CBLs (Zhang et al. 2018), or CMLs (Leba et al. 2012; Zhu et al. 2017) can be involved in regulating plant response to abiotic and biotic stress, consistent with the role of $\mathrm{Ca}^{2+}$ as a ubiquitous second signaling and its role in the crosstalk among plant response to various stresses (Fujita et al. 2006). However, to our knowledge, this is the first report that the CBL family members play a role in the crosstalk between plant immunity and heat stress response.

As a positive regulator of pepper immunity against $R$. solanacearum, the transcriptional regulation of $C a C B L 1$ by RSI suggests that the pepper immunity mediated by $C a C B L 1$ is regulated largely at the transcriptional level. Our data also showed that CaWRKY40 targets the promoter of $C a C B L 1$ via the W-box-2 but not the W-box-1 and this targeting appear to be selective, since, by ChIP-PCR, CaWRKY27 and CaWRKY58, the two other pepper WRKY TFs that also acted as regulators in pepper response to RSI in our previous studies (Dang et al. 2014; Wang et al. 2013), failed to target $C a C B L 1$. However, the possible bindings of these two $\mathrm{W}$ boxes by other WRKY TFs cannot be excluded. The recognition and binding of W-box-2 within promoter of $C a C B L 1$ by CaWRKY40 confers transcriptional regulation of $C a C B L 1$, since $C a W R K Y 40$ silencing significantly downregulated $C a C B L 1$ in pepper plants challenged with RSI/RT, $\mathrm{MgCl}_{2} / \mathrm{HT}$, and RSI/HT. Consistently, the transient overexpression of CaWRKY40-SRDX (Ishida et al. 2007; Ito et al. 2007) also downregulated $C a C B L 1$ in pepper plants. By contrast, transient overexpression of CaWRKY4O significantly enhanced the transcription of $C a C B L 1$ in pepper plants. The direct targeting and positive transcriptional regulation of $C a C B L 1$ by CaWRKY40, in combination with the data that CaWRKY4O was positively regulated by $C a C B L 1$, suggest a feedback loop between $C a C B L 1$ and $C a W R K Y 40$. Feedback loops have been extensively found in transcription networks in plant growth or immunity regulation (Chen et al. 2016; Fan et al. 2014; Lee et al. 2014; Li et al. 2017; Zhang et al. 2009), which might be required for the activation of amplified and prolonged defense signaling upon the challenge of pathogens. Our data showed that the expression of $C a C D P K 15$ was significantly altered by transient overexpression of $C a C B L 1$ or its silencing. We speculate that this might be attributed to CaWRKY40, as CaCDPK15 also acts positively as an upstream regulator of $\mathrm{CaWRKY} 40$ and also forms a positive feedback loop with CaWRKY40 during pepper response to RSI (Shen et al. 2016b). In addition, this result suggests that $\mathrm{Ca}^{2+}$ signaling can be relayed downstream via multiple sensors, which might make the immunity more robust against the attack of pathogens.

Collectively, the data in the present study indicate that $C a C B L 1$ is upregulated by RSI under both RT and HT and acts as a positive regulator in pepper resistance to $R$. solanacearum, forming a positive feedback loop with CaWRKY40.

\section{MATERIALS AND METHODS}

\section{Plant materials and growth conditions.}

Plant materials from the pepper lines HN42, SN, TT5302, and GZ03 as well as $N$. benthamiana were used in this study and the cultivation of these plant materials followed the methods of our previous studies (Cheng et al. 2017; Shen et al. 2016a).

\section{RSI and HT treatment.}

The $R$. solanacearum FJC100301 used in this study was cultivated using the method previously described (Dang et al. 2013; Shen et al. 2016a). Briefly, $R$. solanacearum cells were cultured at $28^{\circ} \mathrm{C}$ overnight and were resuspended with $10 \mathrm{mM} \mathrm{MgCl}_{2}$ solution after centrifugation and adjusted to approximately $10^{8}$ CFU per milliliter. For leaf inoculation, the $R$. solanacearum solution was injected into pepper leaves near the main vein, using a disposable syringe without needle. For root inoculation, roots of pepper plants were mechanically damaged and $5 \mathrm{ml}$ of $R$. solanacearum suspension with an optical density at $600 \mathrm{~nm}$ $\left(\mathrm{OD}_{600}\right)$ of 0.6 were root-irrigated, the pepper plants were placed in an illumination incubator at a temperature of $30^{\circ} \mathrm{C}$ (or $37^{\circ} \mathrm{C}$ ) with $80 \%$ humidity. Disease indices of pepper plants postinoculation were scored from day 1 to day 16 , according to the method described previously (Mukhtar et al. 2008).

\section{The vectors construction.}

To analyze $C a C B L 1$ transient overexpression, the ORF of $C a C B L 1$ with or without termination codon was cloned into the entry vector pDONR207 (Invitrogen) by BP reaction and was then cloned into destination vectors such as pEarleyGate103 (Invitrogen) with a GFP protein tag for subcellular localization or pEarleyGate202 (Invitrogen) harboring a Flag protein tag for ChIP assay by LR reaction.

To investigate the possible binding of CaWRKY40 to W-box-2 within the CaCBL1 promoter, the ORF of CaWRKY4O or its mutant (CaWRKY40-m) was cloned into the vector pDONR207 by BP reaction and was then cloned into destination vector pEarleyGate202 for ChIP assay or pDEST15 (harboring a GST protein tag) for EMSA by LR reaction. 
To generate constructs for a VIGS assay, a specific DNA fragment of $C a C B L 1$ in its ORF was cloned into the entry vector pDONR207 by BP reaction and was then cloned into destination vector pTRV2 (Invitrogen) by LR reaction. The primers used for vector constructions of $C a C B L 1$ and CaWRKY4O in this study are listed in Supplementary Table S1.

The vectors of CabZIP63 and CaWRKY40 used in this study were previously constructed (Shen et al. 2016a). The vectors construction followed Gateway Recombination Cloning Technology (Invitrogen Corp.).

\section{VIGS assay.}

To perform a loss-of-function assay of $C a C B L 1$ in the pepper plant response to RSI under RT or RSI under HT, a tobacco rattle virus (TRV)-based VIGS assay was carried out as previously described (Choi et al. 2012; Shen et al. 2016a). Agrobacterium sp. strain GV3101 constructs harboring TRV1, TRV2:CaCBL1, TRV2:00, and TRV2:CaPDS were grown in Luria-Bertani (LB) broth at $28^{\circ} \mathrm{C}$ overnight. Agrobacterium sp. strain GV3101 cells were resuspended by induction medium (10 mM MES, $10 \mathrm{mM} \mathrm{MgCl}$, $200 \mathrm{mM}$ acetosyringone, $\mathrm{pH}=$ 5.4) and were adjusted to $\mathrm{OD}_{600}=0.8$ after centrifugation. Agrobacterium sp. strain GV3101 containing TRV1 constructs were respectively mixed with that containing TRV2:CaCBL1, TRV2:00, or TRV2:CaPDS at a 1:1 ratio and were then infiltrated into cotyledons of 2-week-old pepper plants.

\section{Transient overexpression of CaCBL1 or CaWRKY40 in pepper leaves.}

Transient overexpression of CaCBL1 or CaWRKY4O in pepper leaves was carried out following the method used previously (Shen et al. 2016a). Agrobacterium sp. strain GV3101 harboring the $35 S$ : CaCBL1-Flag, 35S:CaWRKY40-Flag, or 35S:Flag (empty vector used as a control) constructs were grown in LB broth with $50 \mu \mathrm{g}$ of kanamycin per milliliter and $50 \mu \mathrm{g}$ of rifampicin per milliliter at $28^{\circ} \mathrm{C}$ overnight, were then were resuspended by induction medium after centrifugation, and were adjusted to $\mathrm{OD}_{600}=0.8$. The bacterial suspensions were infiltrated into pepper leaves using a disposable syringe without needle. The infiltrated pepper leaves were harvested at the indicated timepoints.

\section{Subcellular localization.}

For subcellular localization analysis, CaCBL1-GFP was transiently overexpressed by Agrobacterium infiltration in $N$. benthamiana plant leaves or pepper plant leaves of TT5302 and HN42 exactly following the method used previously (Shen et al. 2016a and b).

\section{Immunoblot analysis.}

The immunoblot assay used to detect gene expression at the protein level was performed following a method described previously (Choi et al. 2008). Briefly, total proteins were separated by sodium dodecyl sulfate-polyacrylamide gel electrophoresis (PAGE) and were transferred into polyvinylidene fluoride membranes. The membranes were incubated with primary antiFlag antibody at a 1:5,000 dilution. Goat antirabbit immunoglobulin G (Sigma-Aldrich) with horseradish peroxidase conjugate was used as a secondary antibody, diluted at 1:20,000.

To further investigate the protein expression of CaCBL1 in $N$. benthamiana cells, total proteins were extracted from $N$. benthamiana leaves infiltrated with Agrobacterium sp. strain GV3101 carrying 35S:CaCBL1-GFP or 35S:GFP constructs, as described previously (Choi et al. 2008). The proteins of nuclear fractionation and cytoplasm fractionation were extracted using a plant nuclear or cytoplasmic protein extraction kit (BestBio), respectively. The total nuclear or cytoplasmic proteins were subjected to immunoblot analysis. Anti-H3 (Proteintech) and antiheat-shock complex 70 (Proteintech) were used as nuclear and cytosolic protein markers, respectively.

\section{Prokaryotic expression assay and EMSA analysis.}

To detect the binding of CaWRKY40 or the CaWRKY40 mutant (CaWRKY40-m) to W-box-2 within the CaCBL1 promoter, the recombinant proteins of CaWRKY40-GST and CaWRKY40-m-GST were obtained by a prokaryotic expression system. Briefly, the pDEST15 plasmid containing CaWRKY40 or CaWRKY40-m were transformed into E. coli BL21 (DE3). The recombinant proteins were induced with IPTG (isopropyl $\beta$-D-1-thiogalactopyranoside, $1 \mathrm{mM}$ ) at $20^{\circ} \mathrm{C}$ for $12 \mathrm{~h}$.

EMSA was carried out as described previously (Heravi and Altenbuchner 2014). The wild-type or mutated probe was synthesized by PCR using a single-strand primer and another single-strand primer labeled Cy5-labeled oligonucleotides. The recombinant proteins of CaWRKY40-GST or CaWRKY40-mGST were incubated with wild-type or mutated probe, which was labeled $\mathrm{Cy} 5$ fluorochrome, and $5 \times$ binding buffer $(1 \mathrm{M}$

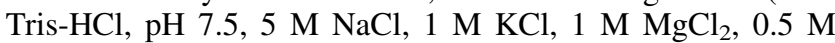
EDTA, pH 8.0, $10 \mathrm{mg}$ of bovine serum albumin per milliliter). The mixture was separated by PAGE gal and was then scanned by Odyssey CLX (LI-COR).

\section{Histochemical staining.}

Leaf staining with trypan blue and DAB was used according to the method described previously (Cai et al. 2015; Choi et al. 2012; Dang et al. 2013; Shen et al. 2016a and b).

\section{qRT-PCR.}

The qRT-PCR assay to analyze the selected gene relative transcription levels was performed as previously described (Cheng et al. 2017; Shen et al. 2016a). The specific primers used in this study are listed in Supplementary Table S1.

\section{ChIP assay.}

The ChIP assay was performed using a method described previously (Qiu et al. 2018). Agrobacterium sp. strain GV3101 cells containing 35S:CaWRKY40-Flag, 35S:CaWRKY58-Flag, or 35S:CaWRKY27-Flag construct were infiltrated into pepper leaves. The infiltrated leaves were harvested at $48 \mathrm{hpi}$, and were then ground in liquid nitrogen. The samples were fixed with $1.0 \%$ formaldehyde for $5 \mathrm{~min}$ and, then $3 \mathrm{M}$ glycine solution was added for $15 \mathrm{~min}$ to terminate the crosslinking reaction. The chromatin was fragmented on ice by probe sonicator to an average length of 300 to $500 \mathrm{bp}$. The protein A agarose beads with anti-Flag antibody (Sigma-Aldrich) were incubated with extracts overnight at $4^{\circ} \mathrm{C}$. After immunoprecipitation, the cross-linking was reversed and the DNA was recovered by purification with phenol/chloroform/isoamyl alcohol (25:24:1). The purified DNA was analyzed by ChIP-PCR using genespecific primers (Supplementary Table S1).

\section{ACKNOWLEDGMENTS}

We thank M. D. Curtis for kindly providing the Gateway destination vectors and S. P. Dinesh-Kumar (Yale University) for the pTRV1 and pTRV2 vectors. We also thank Z. Wang (Fujian Agricultural and Forestry University, China) for providing the pEarleyGate202 plasmids.

\section{AUTHOR-RECOMMENDED INTERNET RESOURCES}

Gateway Recombination Cloning Technology: https://www.thermofisher. com/us/en/home/life-science/cloning/gateway-cloning/gateway-technology PlantCARE online tools: http://bioinformatics.psb.ugent.be/webtools/plant care/html

PSORT online tool: https://www.genscript.com/psort.html SMART website: http://smart.embl-heidelberg.de 


\section{LITERATURE CITED}

Asano, T., Hayashi, N., Kobayashi, M., Aoki, N., Miyao, A., Mitsuhara, I., Ichikawa, H., Komatsu, S., Hirochika, H., Kikuchi, S., and Ohsugi, R. 2012. A rice calcium-dependent protein kinase OsCPK12 oppositely modulates salt-stress tolerance and blast disease resistance. Plant J. 69:26-36.

Batistič, O., Waadt, R., Steinhorst, L., Held, K., and Kudla, J. 2010. CBLmediated targeting of CIPKs facilitates the decoding of calcium signals emanating from distinct cellular stores. Plant J. 61:211-222.

Bhattacharjee, S., Garner, C. M., and Gassmann, W. 2013. New clues in the nucleus: Transcriptional reprogramming in effector-triggered immunity. Front. Plant Sci. 4:364.

Bhattarai, K. K., Atamian, H. S., Kaloshian, I., and Eulgem, T. 2010. WRKY72-type transcription factors contribute to basal immunity in tomato and Arabidopsis as well as gene-for-gene resistance mediated by the tomato $R$ gene Mi-1. Plant J. 63:229-240.

Birkenbihl, R. P., Diezel, C., and Somssich, I. E. 2012. Arabidopsis WRKY33 is a key transcriptional regulator of hormonal and metabolic responses toward Botrytis cinerea infection. Plant Physiol. 159:266-285.

Birkenbihl, R. P., Kracher, B., Roccaro, M., and Somssich, I. E. 2017. Induced genome-wide binding of three Arabidopsis WRKY transcription factors during early MAMP-triggered immunity. Plant Cell 29:20-38.

Buscaill, P., and Rivas, S. 2014. Transcriptional control of plant defence responses. Curr. Opin. Plant Biol. 20:35-46.

Cai, H., Yang, S., Yan, Y., Xiao, Z., Cheng, J., Wu, J., Qiu, A., Lai, Y., Mou, S., Guan, D., Huang, R., and He, S. 2015. CaWRKY6 transcriptionally activates CaWRKY40, regulates Ralstonia solanacearum resistance, and confers high-temperature and high-humidity tolerance in pepper. J. Exp. Bot. 66:3163-3174.

Chen, H. Y., Hsieh, E. J., Cheng, M. C., Chen, C. Y., Hwang, S. Y., and Lin, T. P. 2016. ORA47 (octadecanoid-responsive AP2/ERF-domain transcription factor 47) regulates jasmonic acid and abscisic acid biosynthesis and signaling through binding to a novel cis-element. New Phytol. 211:599-613.

Chen, X., Liu, J., Lin, G., Wang, A., Wang, Z., and Lu, G. 2013. Overexpression of AtWRKY28 and AtWRKY75 in Arabidopsis enhances resistance to oxalic acid and Sclerotinia sclerotiorum. Plant Cell Rep. 32:1589-1599.

Cheng, W., Xiao, Z., Cai, H., Wang, C., Hu, Y., Xiao, Y., Zheng, Y., Shen, L., Yang, S., Liu, Z., Mou, S., Qiu, A., Guan, D., and He, S. 2017. A novel leucine-rich repeat protein, CaLRR51, acts as a positive regulator in the response of pepper to Ralstonia solanacearum infection. Mol. Plant Pathol. 18:1089-1100.

Cheong, Y. H., Pandey, G. K., Grant, J. J., Batistic, O., Li, L., Kim, B. G., Lee, S. C., Kudla, J., and Luan, S. 2007. Two calcineurin B-like calcium sensors, interacting with protein kinase CIPK23, regulate leaf transpiration and root potassium uptake in Arabidopsis. Plant J. 52:223-239.

Choi, D. S., Hwang, I. S., and Hwang, B. K. 2012. Requirement of the cytosolic interaction between PATHOGENESIS-RELATED PROTEIN10 and LEUCINE-RICH REPEAT PROTEIN1 for cell death and defense signaling in pepper. Plant Cell 24:1675-1690.

Choi, H. W., Lee, B. G., Kim, N. H., Park, Y., Lim, C. W., Song, H. K., and Hwang, B. K. 2008. A role for a menthone reductase in resistance against microbial pathogens in plants. Plant Physiol. 148:383-401.

Dang, F., Wang, Y., She, J., Lei, Y., Liu, Z., Eulgem, T., Lai, Y., Lin, J., Yu, L., Lei, D., Guan, D., Li, X., Yuan, Q., and He, S. 2014. Overexpression of CaWRKY27, a subgroup IIe WRKY transcription factor of Capsicum аппиит, positively regulates tobacco resistance to Ralstonia solanacearum infection. Physiol. Plant. 150:397-411.

Dang, F. F., Wang, Y. N., Yu, L., Eulgem, T., Lai, Y., Liu, Z. Q., Wang, X., Qiu, A. L., Zhang, T. X., Lin, J., Chen, Y. S., Guan, D. Y., Cai, H. Y., Mou, S. L., and He, S. L. 2013. CaWRKY40, a WRKY protein of pepper, plays an important role in the regulation of tolerance to heat stress and resistance to Ralstonia solanacearum infection. Plant Cell Environ. 36:757-774.

de la Torre, F., Gutiérrez-Beltrán, E., Pareja-Jaime, Y., Chakravarthy, S., Martin, G. B., and del Pozo, O. 2013. The tomato calcium sensor Cbl10 and its interacting protein kinase Cipk6 define a signaling pathway in plant immunity. Plant Cell 25:2748-2764.

Deng, X., Zhou, S., Hu, W., Feng, J., Zhang, F., Chen, L., Huang, C., Luo, Q., He, Y., Yang, G., and He, G. 2013. Ectopic expression of wheat TaCIPK14, encoding a calcineurin B-like protein-interacting protein kinase, confers salinity and cold tolerance in tobacco. Physiol. Plant. 149:367-377.

Do, H. M., Lee, S. C., Jung, H. W., Sohn, K. H., and Hwang, B. K. 2004. Differential expression and in situ localization of a pepper defensin (CADEF 1) gene in response to pathogen infection, abiotic elicitors and environmental stresses in Capsicum аппиит. Plant Sci. 166:1297-1305.
Fan, M., Bai, M. Y., Kim, J. G., Wang, T., Oh, E., Chen, L., Park, C. H., Son, S. H., Kim, S. K., Mudgett, M. B., and Wang, Z. Y. 2014. The bHLH transcription factor HBI1 mediates the trade-off between growth and pathogen-associated molecular pattern-triggered immunity in Arabidopsis. Plant Cell 26:828-841.

Fujita, M., Fujita, Y., Noutoshi, Y., Takahashi, F., Narusaka, Y., YamaguchiShinozaki, K., and Shinozaki, K. 2006. Crosstalk between abiotic and biotic stress responses: A current view from the points of convergence in the stress signaling networks. Curr. Opin. Plant Biol. 9:436-442.

Guan, D., Yang, F., Xia, X., Shi, Y., Yang, S., Cheng, W., and He, S. 2018. CaHSL1 acts as a positive regulator of pepper thermotolerance under high humidity and is transcriptionally modulated by CaWRKY40. Front. Plant Sci. 9:1802.

Hein, I., Gilroy, E. M., Armstrong, M. R., and Birch, P. R. 2009. The zigzag-zig in oomycete-plant interactions. Mol. Plant Pathol. 10:547-562.

Heravi, K. M., and Altenbuchner, J. 2014. Regulation of the Bacillus subtilis mannitol utilization genes: Promoter structure and transcriptional activation by the wild-type regulator (MtlR) and its mutants. Microbiology 160:91-101.

$\mathrm{Hu}$, Y., Dong, Q., and Yu, D. 2012. Arabidopsis WRKY46 coordinates with WRKY70 and WRKY53 in basal resistance against pathogen Pseudomonas syringae. Plant Sci. 185-186:288-297.

Ishida, T., Hattori, S., Sano, R., Inoue, K., Shirano, Y., Hayashi, H., Shibata, D., Sato, S., Kato, T., Tabata, S., Okada, K., and Wada, T. 2007. Arabidopsis TRANSPARENT TESTA GLABRA2 is directly regulated by R2R3 MYB transcription factors and is involved in regulation of GLABRA2 transcription in epidermal differentiation. Plant Cell 19: 2531-2543.

Ito, T., Nagata, N., Yoshiba, Y., Ohme-Takagi, M., Ma, H., and Shinozaki, K. 2007. Arabidopsis MALE STERILITY1 encodes a PHD-type transcription factor and regulates pollen and tapetum development. Plant Cell 19:3549-3562.

Jambunathan, N., Siani, J. M., and McNellis, T. W. 2001. A humiditysensitive Arabidopsis copine mutant exhibits precocious cell death and increased disease resistance. Plant Cell 13:2225-2240.

Jones, J. D., and Dangl, J. L. 2006. The plant immune system. Nature 444: 323-329.

Kalde, M., Barth, M., Somssich, I. E., and Lippok, B. 2003. Members of the Arabidopsis WRKY group III transcription factors are part of different plant defense signaling pathways. Mol. Plant-Microbe Interact 16: 295-305.

Kim, B. G., Waadt, R., Cheong, Y. H., Pandey, G. K., Dominguez-Solis, J. R., Schültke, S., Lee, S. C., Kudla, J., and Luan, S. 2007. The calcium sensor CBL10 mediates salt tolerance by regulating ion homeostasis in Arabidopsis. Plant J. 52:473-484.

Kissoudis, C., Sunarti, S., van de Wiel, C., Visser, R. G., van der Linden, C. G., and Bai, Y. 2016. Responses to combined abiotic and biotic stress in tomato are governed by stress intensity and resistance mechanism. J. Exp. Bot. 67:5119-5132.

Kolukisaoglu, Ü., Weinl, S., Blazevic, D., Batistic, O., and Kudla, J. 2004. Calcium sensors and their interacting protein kinases: Genomics of the Arabidopsis and rice CBL-CIPK signaling networks. Plant Physiol. 134: 43-58.

Kudla, J., Batistic, O., and Hashimoto, K. 2010. Calcium signals: The lead currency of plant information processing. Plant Cell 22:541-563.

Kudla, J., Xu, Q., Harter, K., Gruissem, W., and Luan, S. 1999. Genes for calcineurin B-like proteins in Arabidopsis are differentially regulated by stress signals. Proc. Natl. Acad. Sci. U.S.A. 96:4718-4723.

Lafortune, D., Béramis, M., Daubèze, A. M., Boissot, N., and Palloix, A. 2005. Partial resistance of pepper to bacterial wilt is oligogenic and stable under tropical conditions. Plant Dis. 89:501-506.

Leba, L. J., Perochon, A., Cheval, C., Ranty, B., Galaud, J. P., and Aldon, D. 2012. CML9, a multifunctional Arabidopsis thaliana calmodulin-like protein involved in stress responses and plant growth? Plant Signal. Behav. 7:1121-1124.

Lee, S., Lee, H. J., Huh, S. U., Paek, K. H., Ha, J. H., and Park, C. M. 2014. The Arabidopsis NAC transcription factor NTL4 participates in a positive feedback loop that induces programmed cell death under heat stress conditions. Plant Sci. 227:76-83.

Li, F., Li, M., Wang, P., Cox, K. L., Jr., Duan, L., Dever, J. K., Shan, L., Li, Z., and He, P. 2017. Regulation of cotton (Gossypium hirsutum) drought responses by mitogen-activated protein (MAP) kinase cascade-mediated phosphorylation of GhWRKY59. New Phytol. 215:1462-1475.

Li, S., Fu, Q., Chen, L., Huang, W., and Yu, D. 2011. Arabidopsis thaliana WRKY25, WRKY26, and WRKY33 coordinate induction of plant thermotolerance. Planta 233:1237-1252.

Li, S., Fu, Q., Huang, W., and Yu, D. 2009. Functional analysis of an Arabidopsis transcription factor WRKY25 in heat stress. Plant Cell Rep. 28:683-693. 
Li, S., Zhou, X., Chen, L., Huang, W., and Yu, D. 2010. Functional characterization of Arabidopsis thaliana WRKY39 in heat stress. Mol. Cells 29:475-483.

Liu, P., Guo, J., Zhang, R., Zhao, J., Liu, C., Qi, T., Duan, Y., Kang, Z., and Guo, J. 2019. TaCIPK10 interacts with and phosphorylates TaNH2 to activate wheat defense responses to stripe rust. Plant Biotechnol. J. 17: 956-968.

Liu, S., Kracher, B., Ziegler, J., Birkenbihl, R. P., and Somssich, I. E. 2015a. Negative regulation of ABA signaling by WRKY33 is critical for Arabidopsis immunity towards Botrytis cinerea 2100 . eLife 4:e07295.

Liu, W. Z., Deng, M., Li, L., Yang, B., Li, H., Deng, H., and Jiang, Y. Q. 2015b. Rapeseed calcineurin B-like protein CBL4, interacting with CBL-interacting protein kinase CIPK24, modulates salt tolerance in plants. Biochem. Biophys. Res. Commun. 467:467-471.

Luan, S. 2009. The CBL-CIPK network in plant calcium signaling. Trends Plant Sci. 14:37-42.

Luo, Q., Wei, Q., Wang, R., Zhang, Y., Zhang, F., He, Y., Zhou, S., Feng, J., Yang, G., and He, G. 2017. BdCIPK31, a calcineurin B-like proteininteracting protein kinase, regulates plant response to drought and salt stress. Front. Plant Sci. 8:1184

Mähs, A., Steinhorst, L., Han, J. P., Shen, L. K., Wang, Y., and Kudla, J. 2013. The calcineurin B-like $\mathrm{Ca}^{2+}$ sensors CBL1 and CBL9 function in pollen germination and pollen tube growth in Arabidopsis. Mol. Plant 6: 1149-1162.

Mohanta, T. K., Mohanta, N., Mohanta, Y. K., Parida, P., and Bae, H. 2015. Genome-wide identification of calcineurin B-like (CBL) gene family of plants reveals novel conserved motifs and evolutionary aspects in calcium signaling events. BMC Plant Biol. 15:189.

Moore, J. W., Loake, G. J., and Spoel, S. H. 2011. Transcription dynamics in plant immunity. Plant Cell 23:2809-2820.

Mukhtar, M. S., Deslandes, L., Auriac, M. C., Marco, Y., and Somssich, I. E. 2008. The Arabidopsis transcription factor WRKY27 influences wilt disease symptom development caused by Ralstonia solanacearum. Plant J. 56:935-947.

Pandey, R., Pieper, G. H., Ter Beek, A., Vischer, N. O., Smelt, J. P., Manders, E. M., and Brul, S. 2015. Quantifying the effect of sorbic acid, heat and combination of both on germination and outgrowth of Bacillus subtilis spores at single cell resolution. Food Microbiol. 52:88-96.

Pandey, S. P., and Somssich, I. E. 2009. The role of WRKY transcription factors in plant immunity. Plant Physiol. 150:1648-1655.

Qiu, A., Lei, Y., Yang, S., Wu, J., Li, J., Bao, B., Cai, Y., Wang, S., Lin, J., Wang, Y., Shen, L., Cai, J., Guan, D., and He, S. 2018. CaC3H14 encoding a tandem $\mathrm{CCCH}$ zinc finger protein is directly targeted by CaWRKY40 and positively regulates the response of pepper to inoculation by Ralstonia solanacearum. Mol. Plant Pathol. 19:2221-2235.

Sanyal, S. K., Kanwar, P., Yadav, A. K., Sharma, C., Kumar, A., and Pandey, G. K. 2017. Arabidopsis CBL interacting protein kinase 3 interacts with $\mathrm{ABR} 1$, an APETALA2 domain transcription factor, to regulate ABA responses. Plant Sci. 254:48-59.

Shen, L., Liu, Z., Yang, S., Yang, T., Liang, J., Wen, J., Liu, Y., Li, J., Shi, L., Tang, Q., Shi, W., Hu, J., Liu, C., Zhang, Y., Lin, W., Wang, R., Yu, H., Mou, S., Hussain, A., Cheng, W., Cai, H., He, L., Guan, D., Wu, Y., and He, S. 2016a. Pepper CabZIP63 acts as a positive regulator during Ralstonia solanacearum or high temperature-high humidity challenge in a positive feedback loop with CaWRKY40. J. Exp. Bot. 67: 2439-2451.

Shen, L., Yang, S., Yang, T., Liang, J., Cheng, W., Wen, J., Liu, Y., Li, J., Shi, L., Tang, Q., Shi, W., Hu, J., Liu, C., Zhang, Y., Mou, S., Liu, Z., Cai, H., He, L., Guan, D., Wu, Y., and He, S. 2016b. CaCDPK15 positively regulates pepper responses to Ralstonia solanacearum inoculation and forms a positive-feedback loop with CaWRKY40 to amplify defense signaling. Sci. Rep. 6:22439.

Tang, R. J., Yang, Y., Yang, L., Liu, H., Wang, C. T., Yu, M. M., Gao, X. S., and Zhang, H. X. 2014. Poplar calcineurin B-like proteins PtCBL10A and PtCBL10B regulate shoot salt tolerance through interaction with PtSOS2 in the vacuolar membrane. Plant Cell Environ. 37:573-588.

Tsuda, K., and Katagiri, F. 2010. Comparing signaling mechanisms engaged in pattern-triggered and effector-triggered immunity. Curr. Opin. Plant Biol. 13:459-465.

Tsuda, K., Sato, M., Stoddard, T., Glazebrook, J., and Katagiri, F. 2009. Network properties of robust immunity in plants. PLoS Genet. 5: e1000772.

Tsuda, K., and Somssich, I. E. 2015. Transcriptional networks in plant immunity. New Phytol. 206:932-947.

Wang, C., Cai, X., and Zheng, Z. 2005. High humidity represses Cf-4/Avr4and Cf-9/Avr9-dependent hypersensitive cell death and defense gene expression. Planta 222:947-956.
Wang, M., Gu, D., Liu, T., Wang, Z., Guo, X., Hou, W., Bai, Y., Chen, X., and Wang, G. 2007. Overexpression of a putative maize calcineurin Blike protein in Arabidopsis confers salt tolerance. Plant Mol. Biol. 65: 733-746.

Wang, Y., Bao, Z., Zhu, Y., and Hua, J. 2009. Analysis of temperature modulation of plant defense against biotrophic microbes. Mol. PlantMicrobe Interact 22:498-506.

Wang, Y., Dang, F., Liu, Z., Wang, X., Eulgem, T., Lai, Y., Yu, L., She, J., Shi, Y., Lin, J., Chen, C., Guan, D., Qiu, A., and He, S. 2013. CaWRKY58, encoding a group I WRKY transcription factor of Capsicum annuum, negatively regulates resistance to Ralstonia solanacearum infection. Mol. Plant Pathol. 14:131-144.

Wang, Y., Sun, T., Li, T., Wang, M., Yang, G., and He, G. 2016. A CBLinteracting protein kinase TaCIPK2 confers drought tolerance in transgenic tobacco plants through regulating the stomatal movement. PLoS One 11:e0167962.

Xi, Y., Liu, J., Dong, C., and Cheng, Z. M. 2017. The CBL and CIPK gene family in grapevine (Vitis vinifera): Genome-wide analysis and expression profiles in response to various abiotic stresses. Front. Plant Sci. 8:978.

Xiao, S., Brown, S., Patrick, E., Brearley, C., and Turner, J. G. 2003. Enhanced transcription of the Arabidopsis disease resistance genes $R P W 8.1$ and $R P W 8.2$ via a salicylic acid-dependent amplification circuit is required for hypersensitive cell death. Plant Cell 15:33-45.

Yin, X., Wang, Q., Chen, Q., Xiang, N., Yang, Y., and Yang, Y. 2017. Genome-wide identification and functional analysis of the calcineurin Blike protein and calcineurin B-like protein-interacting protein kinase gene families in turnip (Brassica rapa var. rapa). Front. Plant Sci. 8: 1191.

Yoshioka, K., Kachroo, P., Tsui, F., Sharma, S. B., Shah, J., and Klessig, D. F. 2001. Environmentally sensitive, SA-dependent defense responses in the cpr22 mutant of Arabidopsis. Plant J. 26:447-459.

Zandalinas, S. I., Fritschi, F. B., and Mittler, R. 2020. Signal transduction networks during stress combination. J. Exp. Bot.

Zeng, H., Zhang, Y., Zhang, X., Pi, E., and Zhu, Y. 2017. Analysis of EF-hand proteins in soybean genome suggests their potential roles in environmental and nutritional stress signaling. Front. Plant Sci. 8: 877.

Zhang, C., Bian, M., Yu, H., Liu, Q., and Yang, Z. 2011. Identification of alkaline stress-responsive genes of CBL family in sweet sorghum (Sorghum bicolor L.). Plant Physiol. Biochem. 49:1306-1312.

Zhang, F., Li, L., Jiao, Z., Chen, Y., Liu, H., Chen, X., Fu, J., Wang, G., and Zheng, J. 2016. Characterization of the calcineurin B-Like (CBL) gene family in maize and functional analysis of ZmCBL9 under abscisic acid and abiotic stress treatments. Plant Sci. 253:118-129.

Zhang, H., Yang, B., Liu, W. Z., Li, H., Wang, L., Wang, B., Deng, M., Liang, W., Deyholos, M. K., and Jiang, Y. Q. 2014a. Identification and characterization of CBL and CIPK gene families in canola (Brassica napus L.). BMC Plant Biol. 14:8.

Zhang, L., Du, L., and Poovaiah, B. W. 2014b. Calcium signaling and biotic defense responses in plants. Plant Signal. Behav. 9:e973818.

Zhang, W., Li, J., Tang, Y., Chen, K., Shi, X., Ohnishi, K., and Zhang, Y. 2017. Involvement of NpdA, a putative 2-nitropropane dioxygenase, in the T3SS expression and full virulence in Ralstonia solanacearum OE11. Front. Microbiol. 8:1990.

Zhang, Y., Lv, Y., Jahan, N., Chen, G., Ren, D., and Guo, L. 2018. Sensing of abiotic stress and ionic stress responses in plants. Int. J. Mol. Sci. 19: 3298.

Zhang, Z., Zhang, H., Quan, R., Wang, X. C., and Huang, R. 2009. Transcriptional regulation of the ethylene response factor LeERF2 in the expression of ethylene biosynthesis genes controls ethylene production in tomato and tobacco. Plant Physiol. 150:365-377.

Zhao, J., Sun, Z., Zheng, J., Guo, X., Dong, Z., Huai, J., Gou, M., He, J., Jin, Y., Wang, J., and Wang, G. 2009. Cloning and characterization of a novel CBL-interacting protein kinase from maize. Plant Mol. Biol. 69: 661-674.

Zhou, Y., Cheng, Y., Yang, Y., Li, X., Supriyo, B., Sun, X., and Yang, Y. 2016. Overexpression of $S p C B L 6$, a calcineurin B-like protein of Stipa purpurea, enhanced cold tolerance and reduced drought tolerance in transgenic Arabidopsis. Mol. Biol. Rep. 43:957-966.

Zhu, K., Chen, F., Liu, J., Chen, X., Hewezi, T., and Cheng, Z. M. 2016. Evolution of an intron-poor cluster of the CIPK gene family and expression in response to drought stress in soybean. Sci. Rep. 6: 28225.

Zhu, X., Perez, M., Aldon, D., and Galaud, J. P. 2017. Respective contribution of CML8 and CML9, two arabidopsis calmodulin-like proteins, to plant stress responses. Plant Signal. Behav. 12:e1322246. 